FERMILAB-TM-2477-DI

November 2010

\title{
Accelerator/Experiment Operations - FY 2010
}

\author{
P. Adamson, J. A. Appel, M. Casarsa, R. Coleman, D. Denisov, R. Dixon, C. Escobar, \\ G. Ginther, S. Gruenendahl, D. Harris, S. Henderson, W. Kissel, W. M. Lee, \\ K. McFarland, C. Moore, R. Plunkett,E. Ramberg, P. Schlabach, \\ A. K. Soha, S. Soldner-Rembold, R. Van de Water
}

Edited by J. A. Appel and H. Ramamoorthi

This Technical Memorandum (TM) summarizes the Fermilab accelerator and accelerator experiment operations for FY 2010. It is one of a series of annual publications intended to gather information in one place. In this case, the information concerns the FY 2010 Run II at the Tevatron Collider, the MINOS and MINERvA experiments using the Main Injector Neutrino Beam (NuMI), the MiniBooNE experiment running in the Booster Neutrino Beam (BNB), and the Meson Test Beam (MTest) activities in the $120 \mathrm{GeV}$ external Switchyard beam (SY120).

Each section was prepared by the relevant authors, and was somewhat edited for inclusion in this summary.

Accelerator Operations (J.A. Appel, R. Dixon, S. Henderson)

\section{Tevatron Collider}

The Fermilab accelerators entered the fiscal year recovering from a long shutdown that ended on September 15, 2009. The official shutdown had begun on Monday, June 15, 2009. Startup after the shutdown was remarkably smooth given the length of the shutdown. By October 1 luminosity was being delivered regularly and initial luminosities soon climbed to the $300 \times 10^{30}$ $\mathrm{cm}^{-2} \mathrm{~s}^{-1}$ level. Downtimes were minor as the Tevatron averaged 123 store hours per running week for the entire year. However we began to experience difficulties internal to the Pelletron. Repairs were difficult, requiring access into the Pelletron tank, which imposes a 36-hour period during which electron cooling is not available. During such periods the initial luminosity of the stores is reduced by approximately $50 \%$. Similar problems recurred several times during the course of the year.

During the first half of the year a large effort was made to rebuild one of the $\mathrm{H}^{-}$columns and to get the source that feeds it working. This source had failed in the previous fiscal year, leaving us without a hot spare. Repairs were completed in March, and the source began running reliably again. Meanwhile, the Tevatron ran very well through the entire period and, during April 2010, achieved a record initial luminosity greater than $400 \times 10^{30} \mathrm{~cm}^{-2} \mathrm{~s}^{-1}$. The high initial luminosities resulted in lower luminosity lifetime and increased dead time in the detectors. As a result, the operating strategy was changed. The best strategy for delivering luminosity to the experiments was to reduce the initial luminosities. Consequently, the initial luminosities were intentionally lowered and the integrated luminosity increased. The record luminosity for one week, $73 \mathrm{pb}^{-1}$, was also achieved in April. However, sustained integrated luminosity was best with initial luminosities of about $350 \times 10^{30} \mathrm{~cm}^{-2} \mathrm{~s}^{-1}$. Early in June, the record annual integrated luminosity, $1.9 \mathrm{fb}^{-1}$ set in FY 2009, was surpassed. Despite the record-level running, problems 
with longitudinal-emittance blowups remained in the Tevatron. The longitudinal emittance of the antiprotons in the Tevatron tended to blow up slightly on many stores. These intermittent problems were not resolved during the year.

The Tevatron ran without major incident until July 18, 2010 when a short maintenance shutdown began. The main objective of the shutdown was to do only necessary maintenance on the complex. Collisions in the Tevatron were re-established on August 21, 2010. The total integrated luminosity for the year up to the shutdown was $2.200 \mathrm{fb}^{-1}$. Another $0.277 \mathrm{fb}^{-1}$ was accumulated after the shutdown and before the end of the fiscal year, bringing the total for the

year to $2.477 \mathrm{fb}^{-1}$, exceeding the previous record fiscal-year luminosity by about $0.5 \mathrm{fb}^{-1}$. The design projection was exceeded by approximately $0.028 \mathrm{fb}^{-1}$.

\section{Commentary on FY 2010 Collider Performance}

The last major Run II Improvement Plan was completed in 2007. Thus, the strategy during the 2010 fiscal year was to maintain reliable operations at the level of performance possible with the accelerator systems available since then.

Highlights for fiscal year 2010 include:

- Average luminosity delivered to CDF and D0 (09/30/09 through 10/01/10) exceeded even the design curve: $2477 \mathrm{pb}^{-1}$ (See Figure 1.)

- Reliability above design goal: average of 123 hours/week of luminosity

- Shutdown initiated as planned July 18, 2010; completed on schedule (4 weeks later plus one week of collider startup)

- Peak initial luminosity range: $75-402 \times 10^{30} \mathrm{~cm}^{-2} \mathrm{sec}^{-1}$

- Weekly integrated luminosity averaged: $52.7 \mathrm{pb}^{-1}$

- Monthly integrated luminosity: $230 \mathrm{pb}^{-1}$

- Peak Antiproton stacking average: 25 - 27x10 $10 / \mathrm{hr}$

- Weekly accumulated antiprotons: 3000 - 3770x10\%10 week

- Unscheduled downtime: $10.6 \%$

- 7631 hours scheduled uptime

- 6825 hours actual uptime

- $\quad$ Significant downtime contributor: Pelletron repairs. 


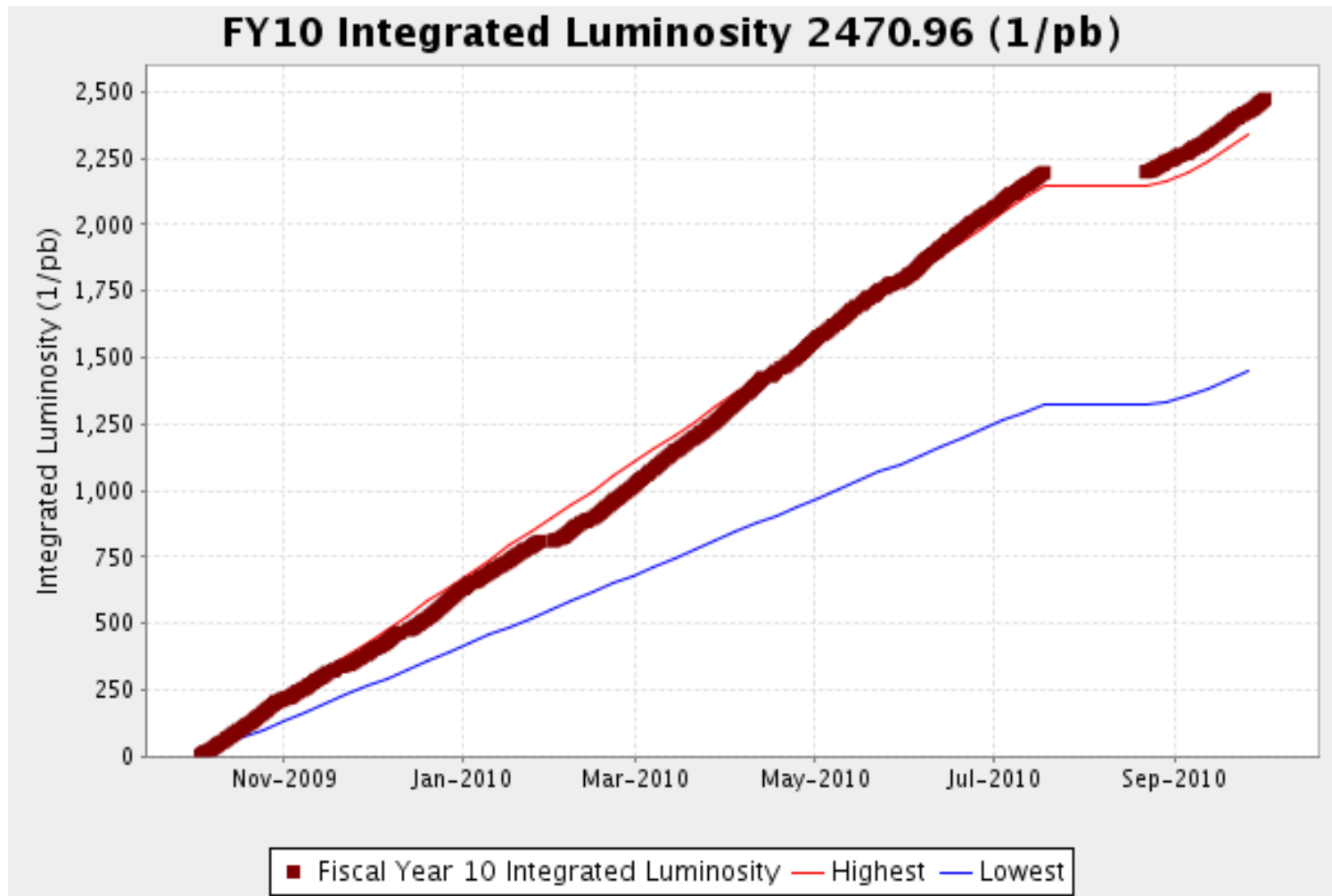

Figure 1. FY 2010 integrated luminosity, planned and delivered (average of CDF and DZero).

In April, after pushing initial luminosities above $4 \times 10^{32} \mathrm{~cm}^{-2} \mathrm{~s}^{-1}$, the Collider operating mode was modified to optimize the integrated luminosity delivered to experiments. The change mitigated the lower luminosity lifetime in the Tevatron, and the increased deadtime in the detectors. The optimization worked well.

Another improvement achieved during the fiscal year was a reduction in the time needed to set up a new store in the Tevatron. This time was reduced from 105 to about 70 minutes with the record being 52 minutes.

Another success of the year was the increase in the length of time between antiproton target failures. During the past two years a new target design was implemented that features a beryllium cover encasing the target. After a few design modifications, a target has now lasted for more than a year. Previous antiproton targets typically lasted 3 to 5 months. Reliability of the antiproton lenses has also increased significantly over the past few years. Lenses now last the better part of a year before they fail. 
Status Relative to the FY 2010 Tevatron Plan

Table 1 shows the planned and actual performance of the Tevatron for the year (defined as from the first Monday of the fiscal year through the first Sunday after the end of the fiscal year).

Table 1. Tevatron Collider planned and actual performance in the Fiscal Year 2010. Note that the Base and Design Profiles given here are the final ones, and are increased goals developed after the start of the fiscal year.

\begin{tabular}{|l|l|l|l|}
\hline & Base Profile & Design profile & Actual $^{*}$ \\
\hline Median initial luminosity $\left(\mathrm{cm}^{-2} \mathrm{~s}^{-1}\right)$ & $1.9 \times 10^{32}$ & $3.4 \times 10^{32}$ & $2.8 \times 10^{32}$ \\
\hline Protons/bunch & $265 \times 10^{9}$ & $295 \times 10^{9}$ & $269 \times 10^{9}$ \\
\hline Pbars/bunch & $43 \times 10^{9}$ & $100 \times 10^{9}$ & $80.0 \times 10^{9}$ \\
\hline Accumulator-Recycler transfer efficiency & $90 \%$ & $96.5 \%$ & $95.5 \%$ \\
\hline Typical peak stacking rate (mA/hour) & 24 & 30 & 26 \\
\hline FY 2010 integrated luminosity (pb $\left.{ }^{-1}\right)$ & 1513 & 2449 & 2477 \\
\hline FY 2010 integrated store time (hours) & 4700 & 5640 & 5782 \\
\hline FY 2010 scheduled uptime (hours) & & & 7631 \\
\hline FY 2010 unscheduled downtime (hours) & & & 806 \\
\hline $\begin{array}{l}\text { FY 2010 unscheduled downtime as } \\
\text { percent of scheduled uptime }\end{array}$ & & & $10.6 \%$ \\
\hline
\end{tabular}

* "Base" and "Design" labels correspond to "end-of-year" goals extended, where appropriate, to the standard reporting periods (Mondays through Sundays). "Actual” values in the first three rows correspond to median values over the entire fiscal year. The goals for Base/Design integrated luminosity per week were $32.2 / 52.1 \mathrm{pb}^{-1}$, and store hours of 100/120 hours/week, for fully scheduled weeks.

\section{$\underline{\text { FY } 2010 \text { Neutrino Operations }}$}

Beam was delivered reliably to the NuMI target for the entire year. Typical beam on target was $3.3 \times 10^{13}$ protons per pulse, and the average power was about $290 \mathrm{~kW}$. A total of $3.2 \times 10^{20}$ protons were delivered to the target during the year (Figure 2), an increase of $45 \%$ relative to the previous year. The most significant downtime resulted from several accesses to repair the dehumidification system. However, in September the NuMI target developed a water leak that eventually led to the failure of the target. This event terminated the NuMI running for the fiscal year. The target had been replaced during the maintenance shutdown, and had only been running a few weeks when the leak developed. Both the MINERvA experiment and the MINOS experiment were impacted by the failure. 


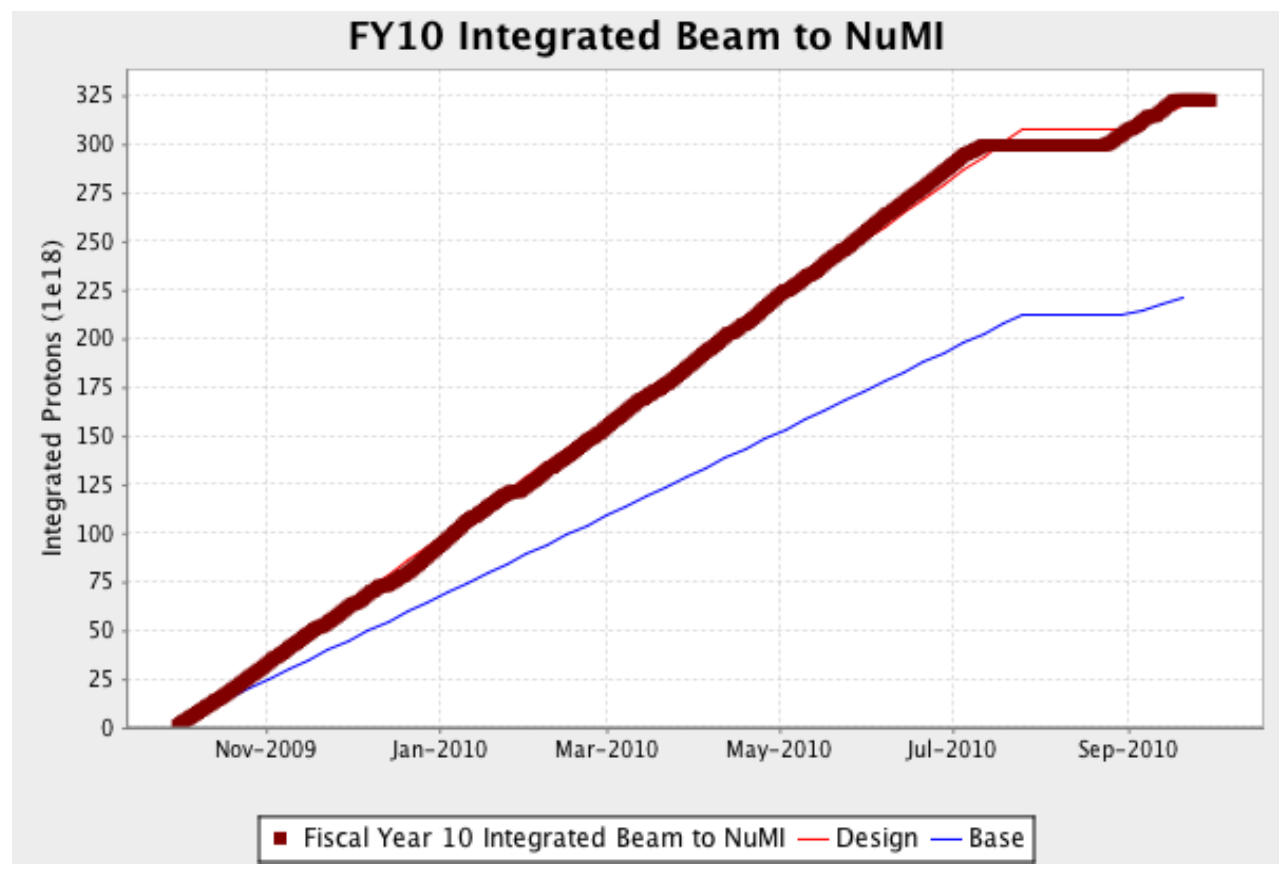

Figure 2. Fiscal Year 2010 integrated proton beam for NuMI

MiniBooNE operations benefited from interruption to NuMI operations, but accumulated most of their $1.7 \times 10^{20}$ delivered protons during the year by running in conjunction with NuMI. MiniBooNE finished the year essentially at their design level. MiniBooNE has accumulated data from a total of $15.0 \times 10^{20}$ protons delivered, and continued to run in anti-neutrino mode at the end of the fiscal year. The $1.7 \times 10^{20}$ protons delivered to the MiniBooNE target during the year (Figure 3) was a $10 \%$ increase over the previous year. The only major interruption was the maintenance shutdown in July and August.

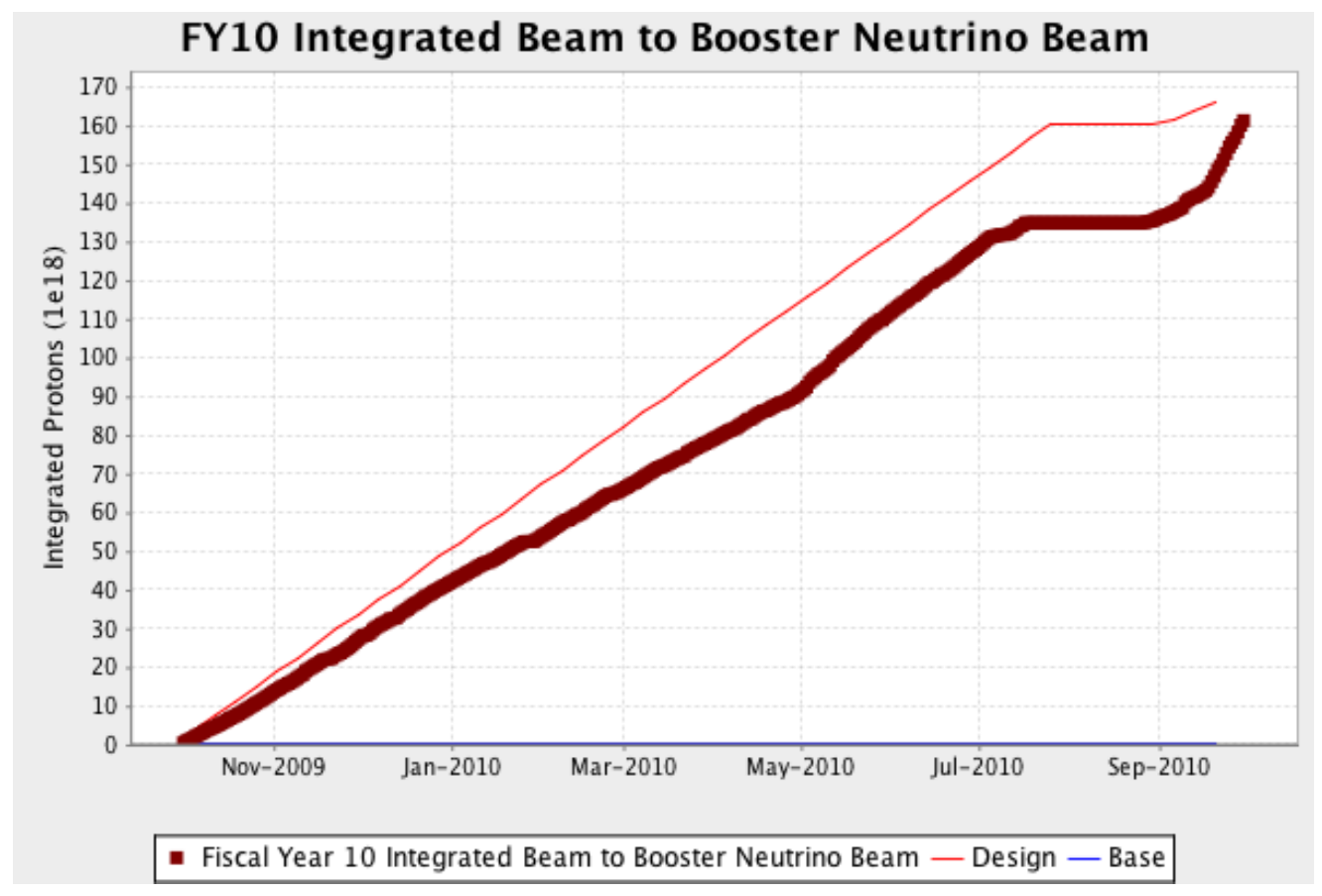

Figure 3. Fiscal year 2010 integrated proton beam for the Booster Neutrino Beam 


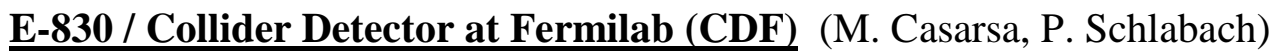

During FY 2010 a total integrated luminosity of $2.5 \mathrm{fb}^{-1}$ was delivered to the CDF detector, of which $2.1 \mathrm{fb}^{-1}$ was recorded to tape. At the end of FY 2010, a total of $9.5 \mathrm{fb}^{-1}$ had been delivered to the experiment in Run II (Figure 4). Overall, the CDF detector has been operating with good live-time and no significant issues, even at the highest instantaneous luminosities. The total data-collection efficiency was $84 \%$, including dead time associated with trigger acceptance, operational inefficiencies (e.g. starting and stopping runs), and downtime from detector problems.

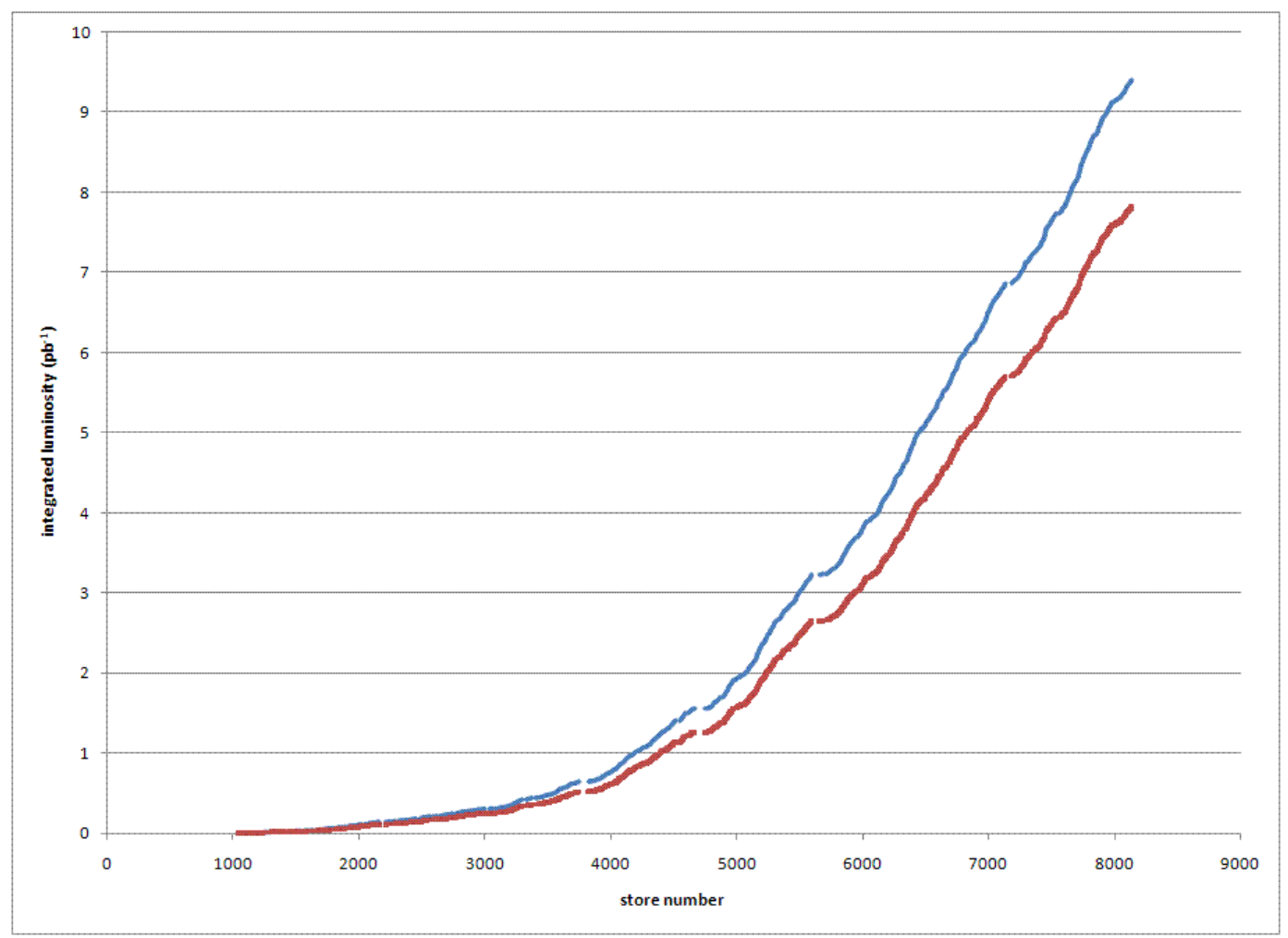

Figure 4. Delivered and recorded luminosity at CDF in Run II.

The Tevatron initial instantaneous luminosities were steadily around $3 \times 10^{32} \mathrm{~cm}^{-2} \mathrm{sec}^{-1}$. The median, average and maximum were 2.8, 2.8 and $4.1 \times 10^{32} \mathrm{~cm}^{-2} \mathrm{sec}^{-1}$, respectively. The CDF trigger is coping well with the higher instantaneous luminosities due to the upgrades of the past several years. CDF commissioned an upgrade to the online displaced vertex trigger. This consolidates track fitting from 16 boards to 1 board on which more modern processors fit tracks faster. The upgrade is intended to increase efficiency and improve purity while potentially allowing lower-impact parameter and transverse momentum cuts and increased acceptance for forward tracks. CDF also increased monitoring of the silicon detector performance as the detector ages, and adjusts detector parameters over time to maintain high efficiency and low noise-hit levels.

As can be seen by the data-taking efficiency shown in Figure 5, after the long summer shutdown which ended shortly before the 2010 fiscal year start, CDF returned quickly to efficient running. 


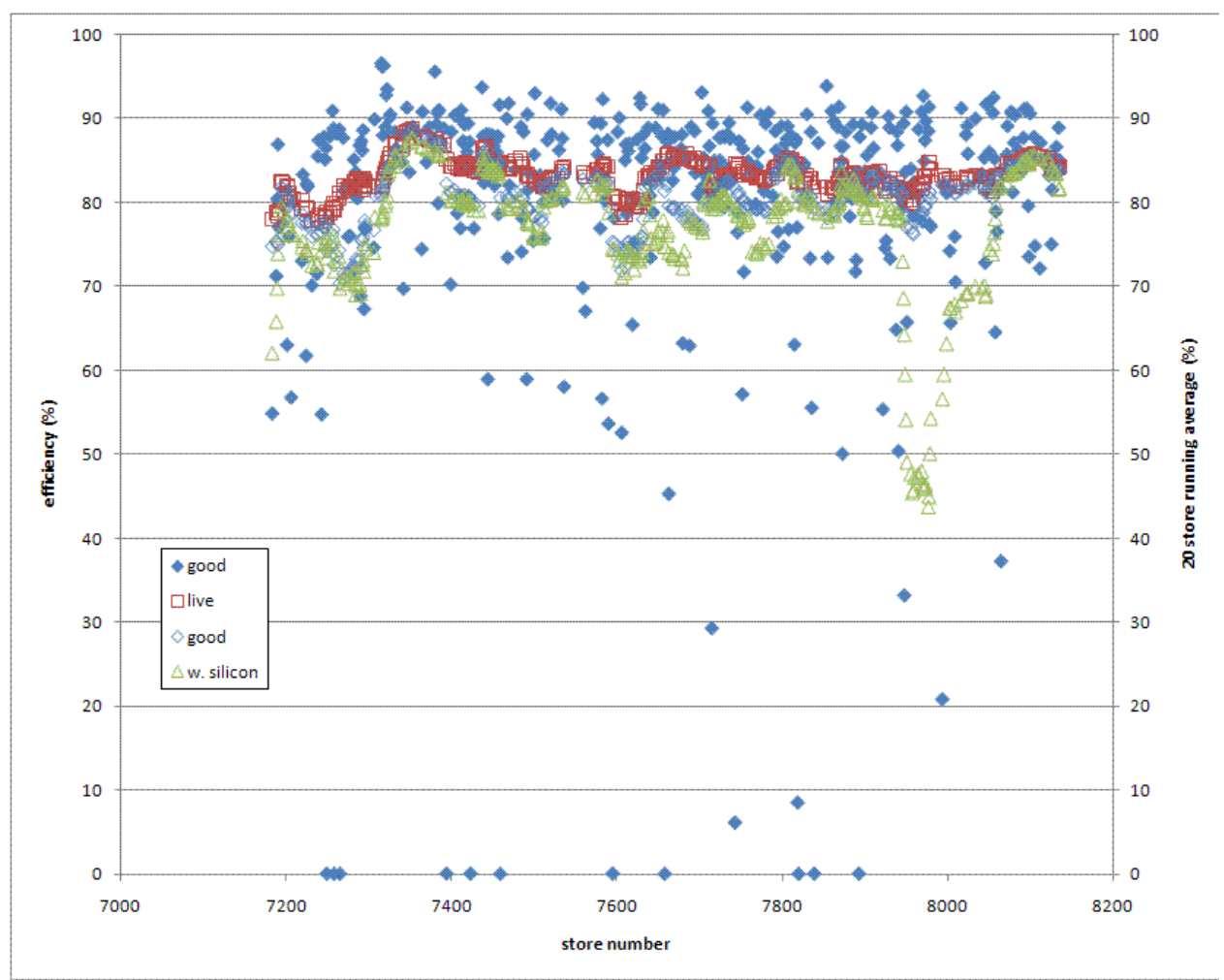

Figure 5. Data-taking efficiency for CDF in fiscal year 2010; blue, run-by-run; green, ten-store running average; and red, thirty-store running average.

During the shutdown a few major jobs were accomplished. CDF opened the End Plugs to repair a short in the COT low voltage bus that had developed in April and to inspect and reseat silicon junction cards. As usual during every shutdown, there were also maintenance and repairs of the HV connections for the COT and refurbishment of silicon power supplies. Two additional ladders of the silicon detector were recovered. The silicon group also made extensive measurements of the light levels being transmitted from the detector. After the shutdown, adjustments were made to balance the signal levels.

Other shutdown work performed included preventive maintenance for the electrical, mechanical, and safety system infrastructure and patching and upgrading software on the online computers. Finally, over 200 people had a chance to visit the hall and admire the detector.

CDF reviewed detector and infrastructure over the course of the summer to evaluate the feasibility of extended running. No "showstoppers" were found.

There were no major changes in shift operations, but there were minor changes to improve the productivity of remote monitoring. CDF continued to move responsibilities around as institutions and people gradually leave. The process is gradual. CDF has moved a few systems per year for the past couple of years, and these adjustments were accomplished again this fiscal year without issue. 
E-823 / DZero (DZero) (D. Denisov, G. Ginther, S. Gruenendahl, W. M. Lee, S. SoldnerRembold)

The accelerator complex delivered an integrated luminosity of $2.46 \mathrm{fb}^{-1}$ to DZero during FY 2010. DZero recorded $2.25 \mathrm{fb}^{-1}$, corresponding to an operating efficiency of $91.6 \%$ during that time period, almost matching the FY 2009 operating efficiency in spite of the higher initial luminosities encountered in FY 2010. Figure 6 illustrates the detector efficiency as a function of time during Run II. The FY 2010 performance represents the best yearly performance to date, exceeding the previous FY record for recorded luminosity at DZero by more than $0.5 \mathrm{fb}^{-1}$. These achievements were made possible by increases in instantaneous and integrated luminosities delivered by the accelerator complex and operational improvements in the detector.

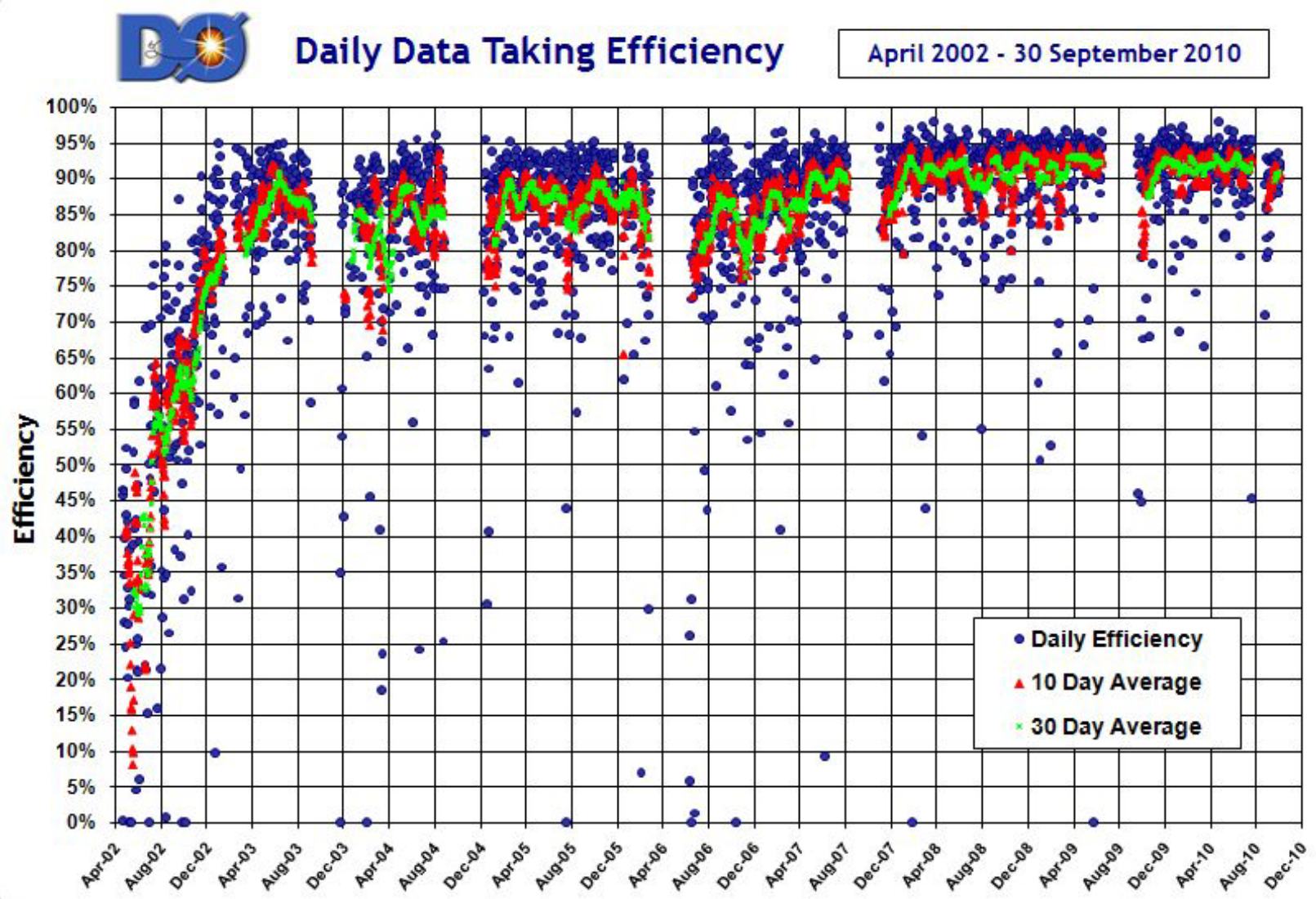

Figure 6. DZero data-taking efficiency as a function of time during Run II. The blue dots represent a daily average, the red triangles represent a 10-day average, and the green squares represent a 30-day average.

DZero continued efforts to streamline operations in the face of increasing instantaneous luminosity, and continued to refine its operations to improve performance. During the past year, streamlining of the begin-store and end-store activities resulted in a reduction in these transition times by about one minute each, contributing to improved efficiency (from the begin-store activities) and increased opportunity to record data (from the end-store related activities). The detector continued to operate reliably, and recorded data at record rates. The operating voltages of the Silicon Microstrip Tracker were adjusted to maintain optimal performance, and monitoring of the impact of radiation damage continued. The results of this monitoring indicate that by the end of FY 2011, as anticipated, it will no longer be possible to fully deplete all of the Layer 1 silicon. Layer 0 was installed during the 2006 shutdown, in part to compensate for this anticipated degradation in performance. In addition to ongoing efforts to address remaining 
sources of dataflow interruptions, there were continuing efforts to improve monitoring tools, and the guidance, training and documentation provided for the shift crews that operate the detector around the clock.

DZero continued to optimize its trigger suite to facilitate routine and efficient operation with initial luminosities above $3 \times 10^{32} \mathrm{~cm}^{-2} \mathrm{~s}^{-1}$. The trigger list is designed to efficiently handle peak luminosities without prescaling high-priority, high- $\mathrm{p}_{\mathrm{t}}$ physics triggers. As of the end of FY 2010, the record initial luminosity at DZero was $4.0 \times 10^{32} \mathrm{~cm}^{-2} \mathrm{~s}^{-1}$.

DZero used the access time during the 4-week-long shutdown in 2010 to perform routine maintenance and introduce additional improvements and refinements. The luminosity monitors were replaced due to radiation damage to the scintillators, and 14 of the 48 photomultiplier tubes were replaced also. Another substantial effort was undertaken to study, and where possible, recover the remaining non-functional Silicon Microstrip Tracker readout channels. More than $1 \%$ of the barrel channels were returned to service, and there were similar gains in the number of operational channels for the detector disks. The readout firmware for the Central Fiber Tracker was updated to reduce readout deadtime. Additional effort was invested in enhancing the performance of the Inter Cryostat Detector by replacing some photomultiplier tubes and highvoltage pods. The muon systems were calibrated, and the firmware was refreshed. The Central Track Trigger equations were re-evaluated to take into account the latest operating conditions and updated alignment input, and studies aimed at further enhancements were made. Safety system tests, single channel repairs, routine and preventive maintenance and infrastructure maintenance (including replacement of a failed chiller) were completed to prepare the detector for operation until the next long shutdown.

As illustrated in Figure 6, the start-up of the DZero detector after the 2010 summer shutdown went very well. The detector was performing well and efficiently collecting high quality data very quickly. Special triggers to gather data for the verification of the calorimeter calibration were collected in parallel with the standard physics suite.

The average operating efficiency of the detector continued its gentle rise, and was $89.2 \%$ averaged over the entire Run II data sample. The integrated luminosity recorded during FY 2010 was 30\% larger than during FY 2009, which was the previous best performance. This new record performance was due to a $\sim 4 \%$ increase in delivered luminosity per operating day, ten fewer weeks of Collider shutdown during FY 2010 compared to FY 2009, and the performance of the DZero detector. As illustrated in Figure 7, the total recorded integrated luminosity increased by $37 \%$ to $8.36 \mathrm{fb}^{-1}$. 
B.8. Run II Integrated Luminosity

19 April 2002 - 30 September 2010

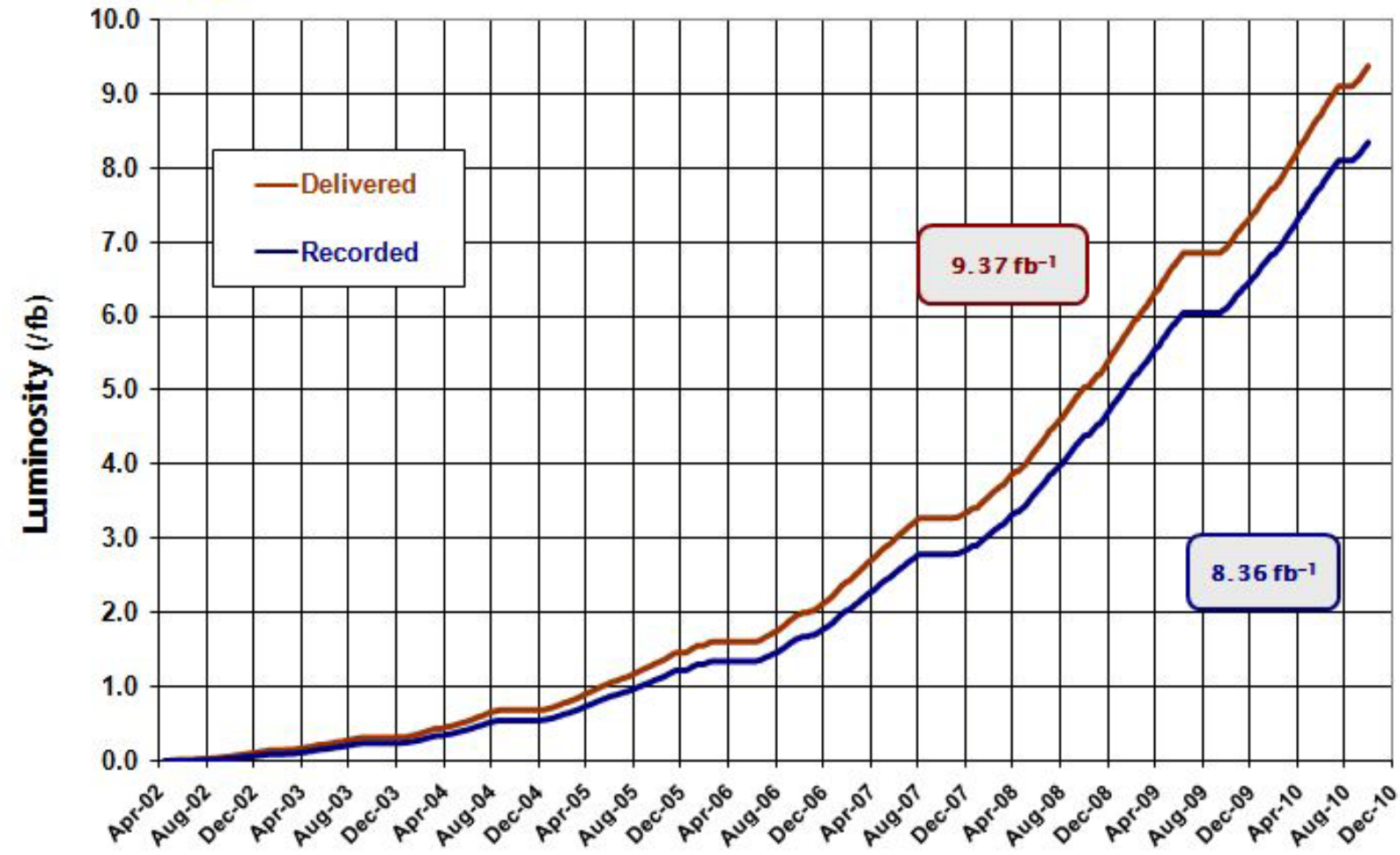

Figure 7. Delivered (red line) and recorded (blue line) luminosity at DZero as a function of time during Run II.

With the improvements in the antiproton stacking rate achieved by the Fermilab Accelerator Division and the ongoing efforts to maintain performance and reduce shot setup time, and in the possible absence of a long shutdown in FY 2011, delivery of an integrated luminosity of more than $12 \mathrm{fb}^{-1}$ to each of the collider detectors before the end of FY 2011 is achievable. DZero continues to publish refereed-journal papers at an average rate of three newresults papers a month. Given the smooth and efficient operation of the DZero detector, the collaboration is well positioned to take full advantage of increasing integrated luminosity and to continue its multifaceted exploration of particle-physics frontiers.

NuMI Beam (P. Adamson, R. Plunkett)

NuMI began FY 2010 having just reversed the polarity of the horn current in order to produce an antineutrino beam. Beam delivery was steady and consistent until the summer shutdown, with an average of $1.05 \times 10^{18}$ protons per day over the period from the start of the fiscal year to the start of the cooldown period (starting July $5^{\text {th) }}$ prior to the summer shutdown. Several new records in beam delivery were achieved during this period - the new record week, with $9.2 \times 10^{18}$ protons delivered, culminating in the new record day, with $1.4 \times 10^{18}$ protons delivered. On March $22^{\text {nd }}$, the horn polarity was reversed, and the facility ran in neutrino mode until the summer shutdown. See Figure 8. 
FY10 NuMI protons to 00:00 Monday 04 October 2010

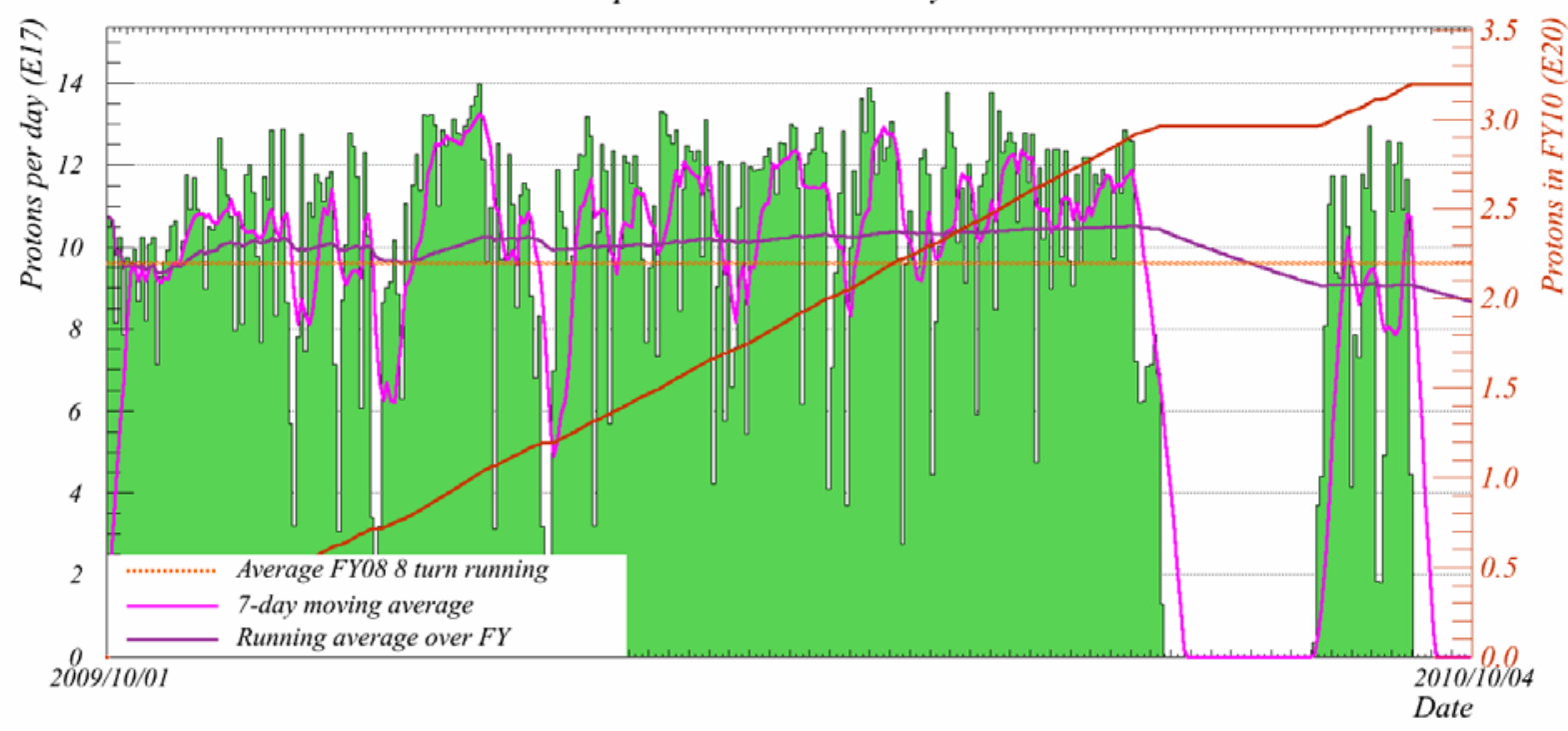

Figure 8. Protons delivered to the target of the NUMI beamline during Fiscal 2010.

On the evening of May $4^{\text {th }}$, the NuMI target NT03 developed a leak in the can that contains its inert helium atmosphere. The hadron and muon monitors showed a step change in response, consistent with a significant change in the length of target in the beam. Low intensity target scans revealed that the target appeared to be bent towards beam-left. The target carrier was rotated to bring the bent target closer to alignment with the beam, and the run was continued until the scheduled summer shutdown. After the target realignment, the neutrino spectrum was not measurably different from the spectrum before the target-bend incident. The target was replaced during the summer shutdown. The failure was located at the ceramic insulator at the upstream end of the target.

Work performed during the summer shutdown:

- During FY 2010, a seal on the target-pile air-circulation-fan-bearing failed. The fan motor and bearing were replaced during the shutdown.

- The C0 radioactive-material storage facility was completed, and the two horns and target NT02 were moved from the NuMI morgue to C0.

- The failed target NT03 was removed and placed in the morgue. NT04 was installed in its place.

- Some beamline quadruple-magnet power supplies were replaced in preparation for NOvA.

Beam returned to NuMI on August $22^{\text {nd }}$, with a series of special runs for MINERvA. The special runs included beam taken in the medium-energy position (LE100) with the horn in both neutrino and antineutrino polarities, and in the high-energy position (LE250) in neutrino mode. A scan of the muon monitor response as a function of horn current was made in both polarities for each target position.

The MINERvA special runs were terminated by a failure of target NT04. This is believed to have begun as a leak in the water cooling lines, which eventually caused a failure in the upstream and downstream target-can beam windows and corrosion of the downstream end of the 
can. Although the target itself does not appear to have been damaged, a target leaking corrosive water is not suitable for installation inside the horn in the LE10 position; so the target was removed beginning on September $17^{\text {th }}$. NuMI remained off for the remainder of the fiscal year while the carrier assembly for target NT05 was completed. A total of $3.2 \times 10^{20}$ protons were delivered to NuMI in FY 2010.

Table 2. FY 2010 NuMI running conditions and proton beam delivered.

\begin{tabular}{|l|l|}
\hline Beam condition & Protons delivered \\
\hline LE010 antineutrinos & $1.74 \times 10^{20}$ \\
\hline LE010 neutrinos & $1.23 \times 10^{20}$ \\
\hline LE100 neutrinos & $9.5 \times 10^{18}$ \\
\hline LE100 antineutrinos & $5.3 \times 10^{18}$ \\
\hline LE250 neutrinos & $8.2 \times 10^{18}$ \\
\hline
\end{tabular}

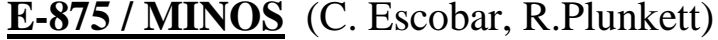

The MINOS experiment continued efficient operation in FY 2010, apart from a period of one week after the summer shutdown, as the Near Detector was turned on. During that week, the humidity in the underground area reached levels around 90\%; with condensation occurring on cooling pipes, causing repeated alarms.

Both Near and Far Detectors operated at extremely high efficiency, 99\% live-time with beam for the statistics-limited Far Detector, and 98\% overall (including scheduled shutdown maintenance). The Near and Far Detectors are robust, requiring only modest ongoing maintenance and upgrades (e.g. replacement of UPS batteries, network upgrades, etc). Typically, the Far Detector has no dead channels, with minor problems repaired rapidly as they appear. The Far Detector required only minimal preventive maintenance during the extended accelerator shutdown, and remained live throughout the summer, collecting cosmic-ray data. In the previous fiscal year, the Far Detector faced persistent network failures. This problem was solved with the installation of new hardware at Soudan. The Near Detector electronics also continued to perform in a steady and consistent fashion, resulting in a similar problem-free readout, apart from the one week period after the summer shutdown, when a higher than usual number of front-end electronics components had to be replaced. The Near Detector downtime was dominated by infrastructure work related to electrical modifications done to allow operation of multiple experiments.

The Near Detector Hall is now shared with other experiments, with a subsequent impact on MINOS Near Detector operations. There is an ongoing training program for the MINERvA Collaboration whereby when a MINOS Near Detector intervention is needed, an attempt is made to always have someone from MINERvA accompany and assist in order to become familiar with the operation of the Near Detector, which is needed for MINERvA operations. 
In FY 2010 MINOS produced preliminary results on the oscillation parameters of muon antineutrinos, using a dedicated beam of NUMI antineutrinos with exposure of $1.7 \times 10^{20}$ protons on target. The resulting measured parameters show some tension with the more precisely measured parameters for muon neutrinos, in contrast to predictions. The result is shown in Figure 9, and was in preparation at the end of the fiscal year for submittal for publication. Additional antineutrino running will reduce the significant statistical uncertainty in the antineutrino result.

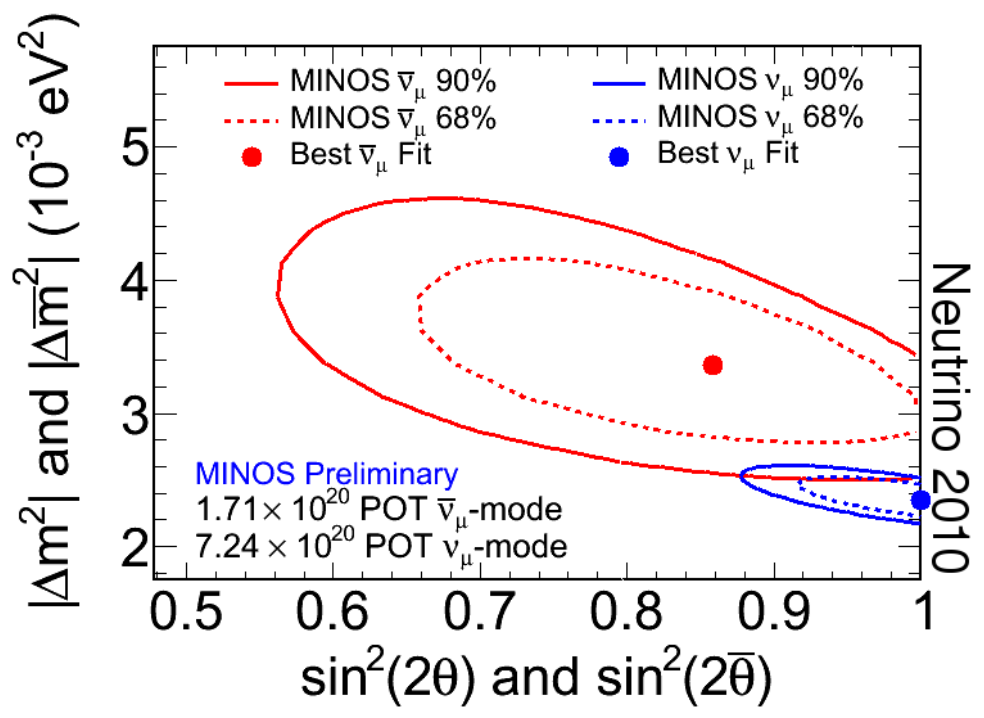

Figure 9. The allowed region in parameter space from the analysis of charged-current antineutrino interactions in the MINOS detector resulting from $1.7 \times 10^{20} \mathrm{NuMI}$ protons on target, compared to the most recent MINOS preliminary results for the neutrino channel.

The MINOS detector was optimized for charged-current neutrino interactions that produce muons. While it is more difficult to observe electrons, it is possible. The first analysis of a search for electron-neutrino interactions in the MINOS Far Detector was published in FY 2010 in Physical Review Letters, 103: 261802 (2009). This past year, the Collaboration completed an updated analysis of electron appearance in the MINOS Far Detector using more data, which was presented at many conference and seminar presentations and published as Phys. Rev. D (Rapid Communications) 82: 051102 (2010). Based on an exposure of $7 \times 10^{20}$ protons on target, MINOS observed $54 \quad v_{\mathrm{e}}$ charged-current events with an expected event background of $49.1 \pm 7.0$ (stat) \pm 2.7 (syst). This enabled MINOS to produce the world’s best limits on this process for most of the range of possible CP violation, for the normal mass hierarchy (Figure 10). 


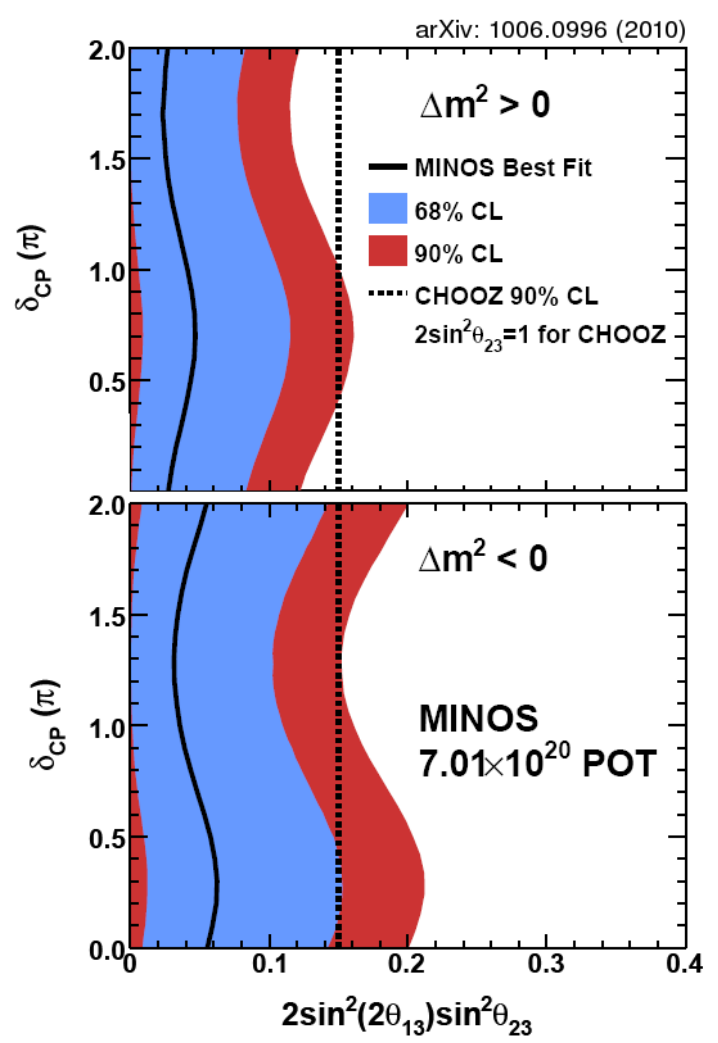

Figure 10. Limits and best fit values obtained by the MINOS experiment for electron-neutrino appearance in the NUMI beam due to the oscillation process. Top (bottom) figure shows the limit compared to expectations for the normal (inverted) mass hierarchy.

Ongoing analysis of the increasing data set continues to improve the knowledge of the $v_{2} \leftrightarrow v_{3}\left|\Delta \mathrm{m}^{2}\right|$ oscillation parameter. The oscillation parameters are now measured to be $\Delta \mathrm{m}^{2}=$ $2.35 \pm 0.13_{-0.08}^{+0.11} \times 10^{-3} \mathrm{eV}^{2}$ and $\sin ^{2} 2 \theta>0.91$ (90\% confidence). MINOS data disfavors nonstandard $v_{\mu}$ disappearance models such as neutrino decay (by $6 \sigma$, or $8 \sigma$ if neutral currents are included) and decoherence (by $8 \sigma$ ). Figure 11 shows the most recent results, which were being prepared for publication at the end of the fiscal year.

To check if the observed $v_{\mu}$ disappearance might involve a fourth, sterile neutrino flavor, the rate of Neutral Current (NC) interactions can be compared between the Near and Far Detectors. Such a sterile neutrino would have no NC interactions, while any of the three known flavors would have such interactions. An exposure of $7.1 \times 10^{20}$ protons-on-target was used to make a comparison of the Far Detector spectrum with expectations derived from the observed high-statistics Near Detector data. The data are consistent with no sterile neutrino participation, with a measurement of the fraction of sterile participation $\mathrm{f}_{\mathrm{s}}<0.22$ at $90 \%$ confidence level. A publication was in preparation at the end of the fiscal year. Earlier analysis of a smaller data set appeared in Phys. Rev. D 81: 052004 (2010). 


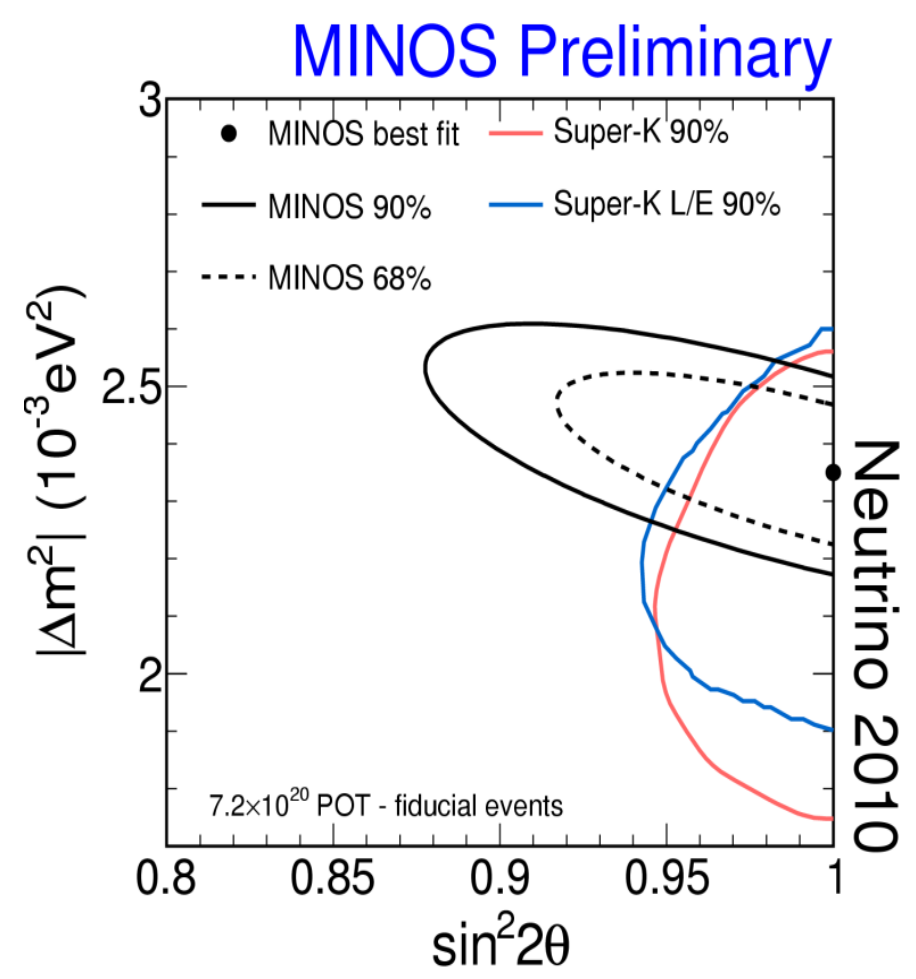

Figure 11. Limits and best fit values obtained by the MINOS experiment for the oscillation parameters of muon neutrinos created in the NUMI beam due to the oscillation process.

In addition to the oscillation-related analyses, MINOS published in FY 2010 a measurement of the total neutrino and antineutrino charged current cross sections, appearing as Phys. Rev. D 81: 072002 (2010), The MINOS far detector data was used to study Lorentz invariance [Phys.Rev. Lett. 105: 151601 (2010)], and a variety of cosmic ray effects were studied in Phys. Rev. D 81: 012001 (2010) and two papers in preparation. An updated study of the oscillation of atmospheric neutrinos is also being prepared. Collaboration members published one technical paper during this period.

E-938 / MINERvA (D. Harris, K. McFarland)

\section{MINER vA Construction and Installation Activities in FY 2010}

The MINERvA Project Construction and Installation was completed in the first quarter of FY 2010. The project was reviewed for CD-4 approval in mid-May 2010, and at the end of June there was an ESAAB meeting where the project was granted CD-4 approval for start of operations. The construction project was completed 1.5M\$ (9\%) under budget, and received CD4 approval three months before the baseline review date.

Because the NuMI beamline was already operational, the experiment began installing the downstream $55 \%$ of the detector in the fall of 2009 with the goal of taking data on the partially completed detector while the last components were being built and commissioned. This 
installation proceeded through early November, and the first physics data taking began in midNovember 2009. Once the last modules were built and calibrated, the remaining modules were installed underground, along with the solid nuclear targets. That installation began in early January 2010 and by mid-March, all 120 detector modules were installed and commissioned. These modules included all of the solid targets used in the MINERvA experiment: graphite, lead, and steel. A short transition period occurred where the data acquisition system for the upstream detector was expanded to include the downstream detector components (which were being read out by the prototype data acquisition system that was retired in favor of the newer, significantly faster final system).

As part of an NSF MRI grant, in mid-FY 2010 the experiment also completed the design and began procurement of an acrylic-based vessel to serve as water target for the neutrino beam. This target was designed to be placed between sequential detector modules in the upstream nuclear target region. There were two prototypes fabricated in the spring to develop the design, and by the middle of the summer 2010 shutdown, the vessel was delivered to Fermilab for tests. After several weeks of testing, the vessel was filled with water. However, before installation, there were small fissures that started to develop in the acrylic vessel. The vessel itself did not leak, and the experiment placed the vessel in the neutrino beam while a new Kevlar and steelbased alternate design was pursued.

In FY 2010, the MINERvA experiment also built and tested a 40-scintillator-plane testbeam detector (T-977). The active component of this detector had scintillator planes that measured $1.07 \mathrm{~m} \times 1.07 \mathrm{~m}$ in transverse size, but were otherwise the same construction as the MINERvA neutrino detector scintillator planes. The 40 planes were mounted on the detector stand in such a way that every other plane was rotated either +60 or -60 degrees compared to the plane before it, similar to the "XUXV" configuration of the full neutrino detector. The construction of the test beam planes proceeded between October 2009 and May 2010, with partial shipments along the way to allow for test beam plane commissioning, similar to the neutrino detector module commissioning. Once all the planes were ready for taking data, the test beam detector itself could be configured in different ways to test the modeling of different parts of the detector. This is done by separating successive planes with 1" thick steel plates to simulate the hadron calorimeter region, or $2 \mathrm{~mm}$ thick lead sheets to simulate the electromagnetic calorimeter. The fully active tracker region of the detector is simply reproduced by taking data with the scintillator planes stacked without absorber.

\section{Physics Goals for MINERvA in Low Energy Beam}

The goal during the low-energy neutrino running is to provide exclusive cross-section measurements on a variety of nuclei. The data will help in understanding the details of neutrino interactions, and serve as important constraints for current and future oscillation experiments. Low energy events tend to have few final state particles - which allows the MINERvA detector to identify single particles and the exclusive channels important for current and future oscillation experiments. MINERvA's detector is about a factor of 10 more fine-grained than the NOvA detector, and can identify things that contribute backgrounds to NOvA. MINERvA sees a higher neutrino beam energy than T2K's near detector, and measures reactions that contribute backgrounds to T2K from the high-energy tail of the beam (where "high energy" in the T2K case 
means above $1 \mathrm{GeV}$ ). Low energy data will also allow a study of exclusive channels as a function of target nuclei. Various processes are expected to have different nuclear dependencies. MINERvA should be able to see those different dependencies clearly if it accumulates the expected amount of data.

\section{Time Line of MINER vA Operations}

MINERvA started taking physics-quality data with its partially completed detector starting in November 2009. For this detector the entire downstream hadron and electromagnetic calorimeters were installed, as well as about half the active target modules. Between November and January there was also one nuclear target containing both iron and lead, and a veto region before the active tracker volume, allowing first nuclear target studies with antineutrinos. After January the nuclear target and veto region were removed to continue installation of the remaining 45\% of the detector. During the second installation period, from January 2010 through March 2010, the downstream detector components remained in a stable configuration and the experiment continued to take antineutrino data.

Between the start of the MINERvA anti-neutrino run until March 1, 2010, the ArgoNeuT Liquid Argon TPC test was located between the MINERvA detector and the MINOS near detector. This represented about 2 metric tons of material (only 0.9 of which was the argon itself) in a complicated geometry which has to be simulated to account for the degradation of the muon momentum measurements in the MINOS detector. Approximately $40 \%$ of the muons that start from the fiducial region of MINERvA and pass into the MINOS near detector traversed some amount of ArgoNeuT, most of which was inactive. The average energy loss passing through ArgoNeuT was about $100 \mathrm{MeV}$, but the variation of the energy loss is large from event to event, and in some regions up to a GeV of energy would be lost between MINOS and MINERvA. ArgoNeuT was drained of the Argon at the end of February, and was removed from between the two detectors on March 1, 2010.

On March 22, 2010 the detector installation and checkout was completed and the neutrino running began. This run includes all of the solid nuclear targets that are planned for the experiment. The livetimes quickly reached the 95\% level and above after about a week of running, and have been stable and high since that time, with the exception of a special run period in August, 2010 after the first shutdown. During the special runs, the MINOS near detector was not live for about $3 \times 10^{18}$ of the protons delivered, due to troubles with both the MINOS near detector electronics and coil. The Low Energy neutrino run lasted until the planned summer shutdown that started for the NuMI beamline on July 13. During this first run period, the NuMI beamline delivered $1.214 \times 10^{20}$ protons on target, and of those, the experiment recorded $1.153 \times 10^{20}$ protons on target. This represents about $29 \%$ of the low energy neutrino exposure on which the physics case for the experiment was made. The following event displays show one of three views for three different events. 

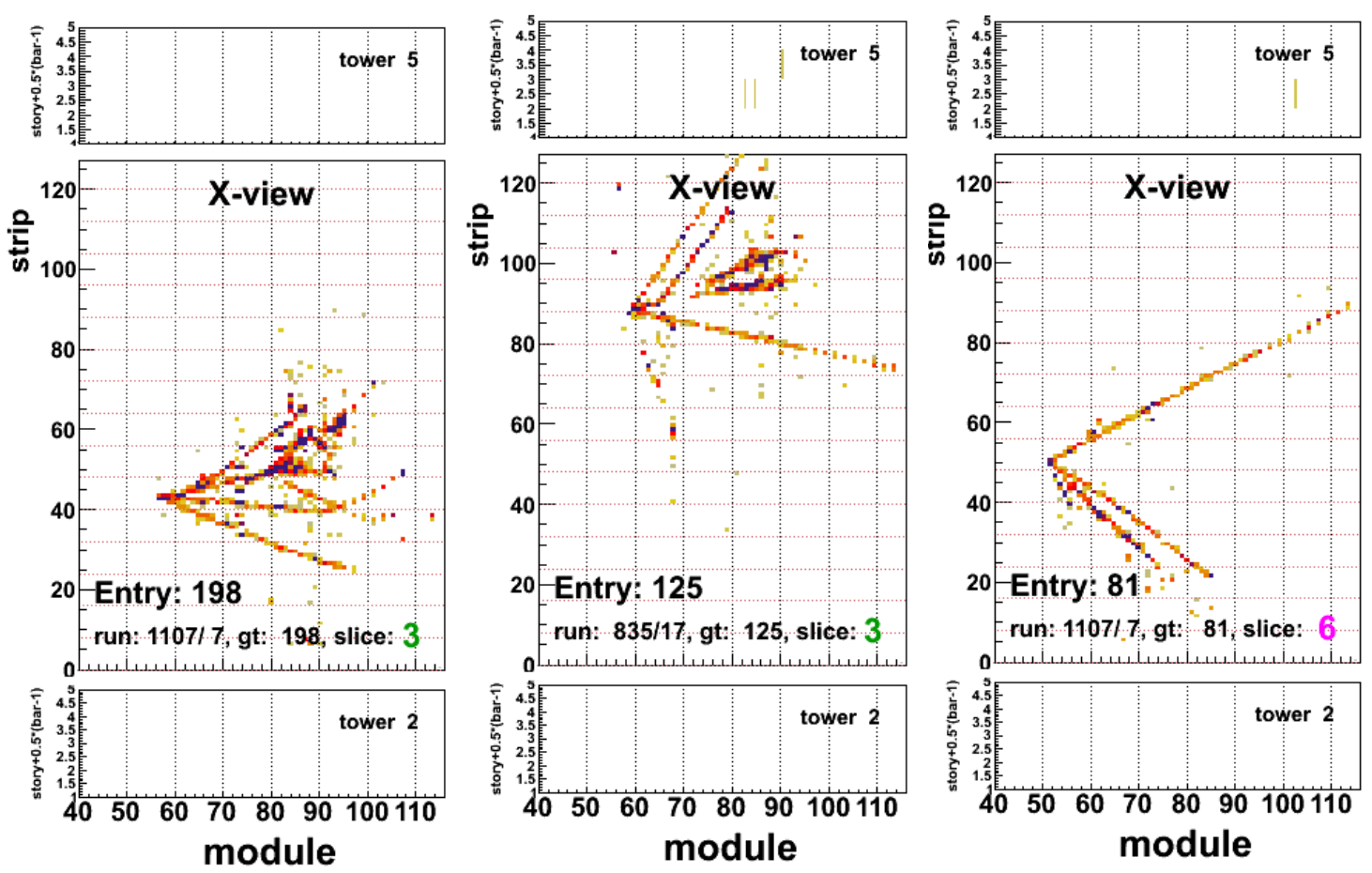

Figure 12. One view of three different events from MINERvA’s neutrino data in FY 2010.

During the summer shutdown, there were a few hardware swaps that were made on the detector to make sure that the best-performing phototubes were the ones that were on the detector. When a final set of spare phototubes arrived in late August, the spares pool was roughly 30 phototubes. The data acquisition system software was also upgraded to allow for the faster data-taking needed after the shutdown for the special runs with higher-energy neutrino beam (which means higher interaction rates).

Also during the summer shutdown, it was determined that the water target would likely last a long time if it were only filled to $75 \%$ of its full capacity, so the empty target was installed. The plan was to run with the partially filled target while an alternate design was pursued, and to collect neutrino data with this first target. Since it was not clear how long the vessel would last, and since the special run data were not going to be used for cross section measurements on water, the plan was to fill the vessel after the special runs were complete.

The neutrino beam resumed on August 23, 2010, after the NuMI target was replaced. Because the NuMI graphite target had just been replaced, it was an opportune time to do the special runs where the target is in a different position relative to the horns. The special neutrino runs were taken with two target positions (medium and high energy), and for both neutrino and antineutrino beams in the medium-energy position. Because of the NuMI target problem described above, the beamline was shut down starting on September 17 for the remainder of FY 2010 while the new spare target was prepared. The statistics that were collected are shown in the following table, where these numbers do not include the beam that was taken the first few days after the summer and a day during the high-energy running when the MINOS near detector was not operational (totaling about $3 \times 10^{18}$ ). 


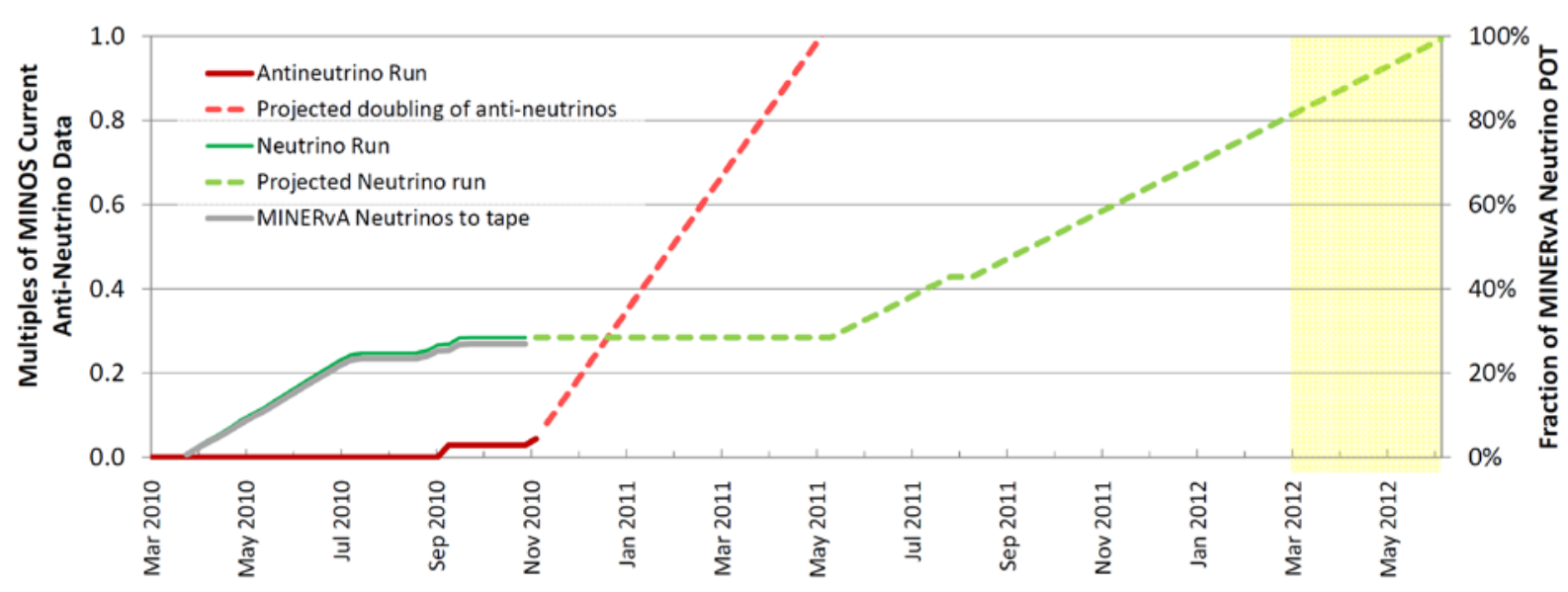

Figure 13 Recent NuMI running, the MINERvA data written to tape, and one possible projected future run. The projection assumes the NuMI complex will repeat its past performance over a one-year uptime average without any future long downtime except for one two-week shutdown for a target change. The red dashed line shows a run, just started, to double the MINOS anti-neutrino exposure. The green line shows a run following the anti-neutrino run to give MINERvA its full requested low-energy neutrino exposure. The shaded yellow area is time after the current date for the shutdown to begin NOvA improvements to the Main Injector (if the Tevatron run is not extended) and to the NuMI beamline.

Table 3. Normal and special runs for the low-energy-target running, as well as the protons recorded in each configuration in FY 2010.

\begin{tabular}{|l|l|l|l|}
\hline Run & Dates & POT Recorded & Goal \\
\hline $\begin{array}{l}\text { Target at 100cm, Forward } \\
\text { Horn Current 200kAmps }\end{array}$ & $\begin{array}{l}\text { August 26 through } \\
\text { September 3 }\end{array}$ & $6.99 \times 10^{18}$ & $15 \times 10^{18}$ \\
\hline $\begin{array}{l}\text { Target at 100cm, Reverse Horn } \\
\text { Current 200kAmps }\end{array}$ & September 3 through 8 & $4.08 \times 10^{18}$ & \\
\hline $\begin{array}{l}\text { Target at 250cm, Forward } \\
\text { Horn Current 200kAmps }\end{array}$ & September 8 through 17 & $7.73 \times 10^{18}$ & $15 \times 10^{18}$ \\
\hline $\begin{array}{l}\text { Target at 10cm, Forward Horn } \\
\text { Current 200kAmps }\end{array}$ & - & 0 & $15 \times 10^{18}$ \\
\hline $\begin{array}{l}\text { Target at 10cm, Forward Horn } \\
\text { Current 150kAmps }\end{array}$ & - & 0 & $15 \times 10^{18}$ \\
\hline $\begin{array}{l}\text { Target at 10cm, 0kAmps, } \\
\text { MINOS coil focusing negative } \\
\text { muons }\end{array}$ & - & 0 & $15 \times 10^{18}$ \\
\hline $\begin{array}{l}\text { Target at 10cm, Forward Horn } \\
\text { Current, 180kAmps (nominal } \\
\text { low-energy running) }\end{array}$ & March 22 through July 12 & $121 \times 10^{18}$ & $400 \times 10^{18}$ \\
\hline
\end{tabular}

In addition to the neutrino and antineutrino special run data that were taken, there were horn current scans in both horn polarities and both target positions that were taken to provide a separate possible constraint on the flux prediction. These current scans require only a few spills at several different horn currents, and each scan takes approximately two hours to collect. Those data will be analyzed to try to further constrain the flux prediction. See the raw data shown in the Figure 14. 
Target $100 \mathrm{~cm}$ upstream of horn

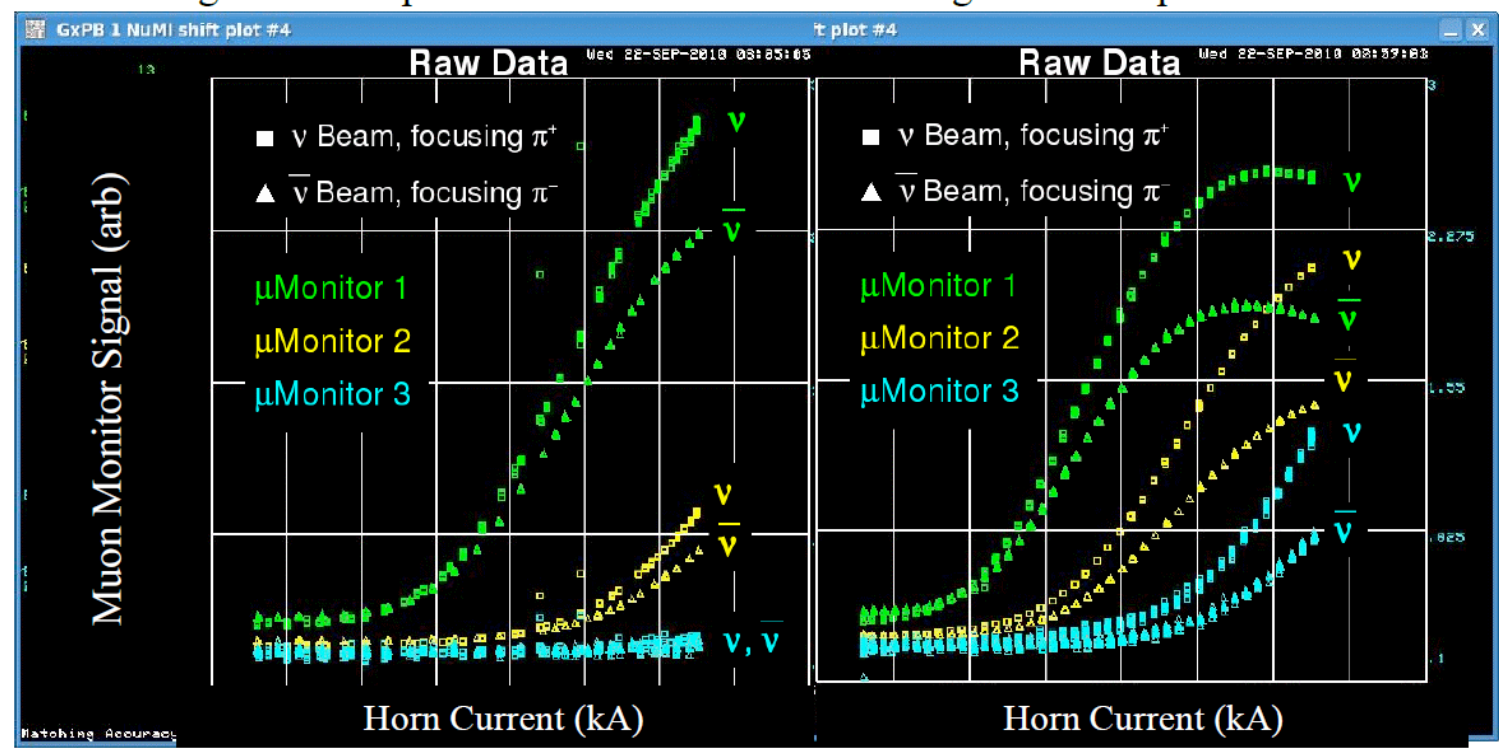

Figure 14. Raw data from the horn current scans that were done when the target was in the medium-energy position (left) and the high-energy position (right).

\section{Operations Summary of Test Beam Experiment (T-977)}

A first commissioning run for the MINERvA detector study in the Meson Laboratory test beam, took place in May in which 8 detector planes were installed and read out, and the test beam detector data acquisition system was integrated with that of the beamline instrumentation. The upstream beam-line instrumentation consisted of 4 drift chambers that were re-furbished from the HyperCP experiment, and a time of flight system that was scintillator-based and used as a trigger. A new tertiary beamline produced particles that ranged between 0.4 and $1.5 \mathrm{GeV}$, much of the range of secondary particles produced in 3-20GeV neutrino interactions.

The MINERvA test beam detector took data between June 7 and June 27 in a configuration where the upstream 20 planes were configured like the electromagnetic calorimeter (ECAL) of MINERvA (with $2 \mathrm{~mm}$ of lead between each plane), and the downstream 20 planes were configured like the hadron calorimeter of MINERvA (with 1" of steel between each plane). Between July 1 and July 16, data were taken with no absorber between the first 20 planes of scintillator, and $2 \mathrm{~mm}$ of lead between the downstream 20 planes to model the tracker-ECAL interface of the neutrino detector. Both positive and negative particle data were taken for both detector configurations. A table of the various triggers recorded is show in Table 4. Of the very loose triggers, roughly $10 \%-12 \%$ are from events with single reconstructible particles in the beamline.

Table 4. Trigger statistics from the test-beam run for the different focusing polarities and the different test beam detector configurations.

\begin{tabular}{|l|l|l|l|}
\hline Configuration & Negative Pion Run & Positive Pion Run & Beam Muon Run \\
\hline 20 Tracker, 20 ECAL & $98.1 \mathrm{k}$ & $13.1 \mathrm{k}$ & $17.5 \mathrm{k}$ \\
\hline 20 ECAL, 20 HCAL & $77.2 \mathrm{k}$ & $73.7 \mathrm{k}$ & $16.2 \mathrm{k}$ \\
\hline
\end{tabular}

A plot of the reconstructed track momentum versus time of flight is shown in the Figure 15. The different populations of positive and negative particles are clearly seen, as are the accidental triggers that arrive at the next rf bucket 20 nanoseconds later. 

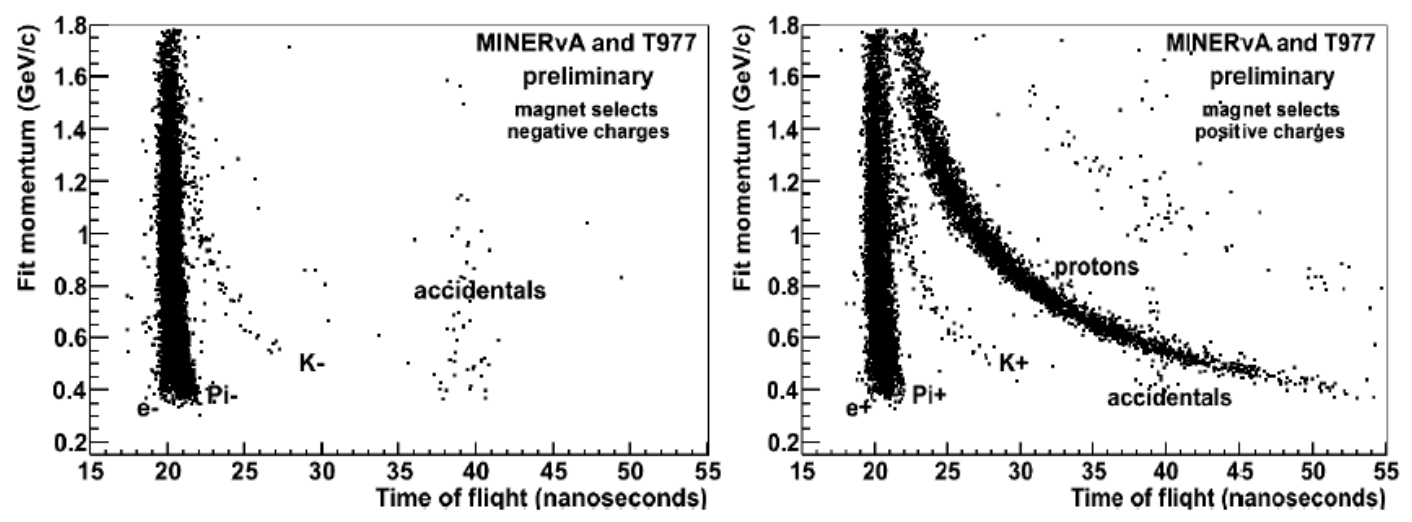

Figure 15. Reconstructed particle momentum versus the time of flight according to the upstream beamline instrumentation in MTest. The different populations of test beam particles are clearly indicated.

The following test beam event display shows a particle that is consistent with being a pion, according to the upstream beamline instrumentation.

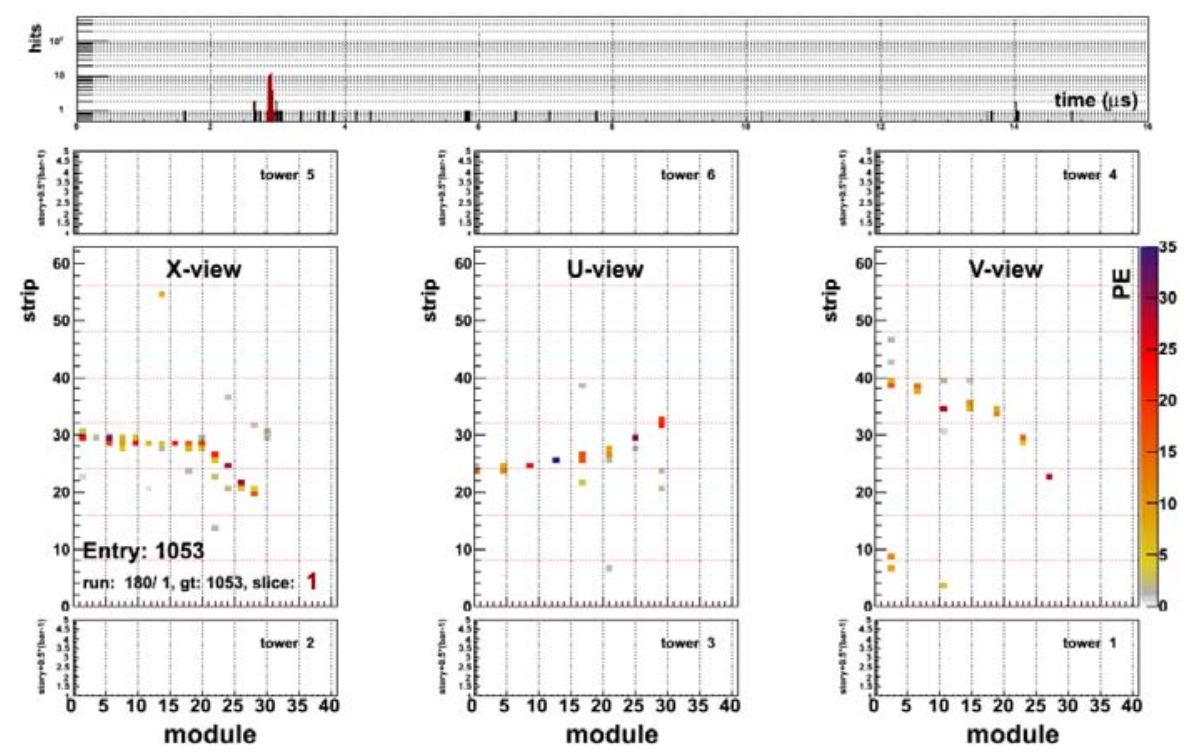

Figure 16. Three views of an event with a pion candidate in the MINERvA test-beam detector.

\section{E-944 / MiniBooNE (R. Van de Water)}

The FY 2010 Booster Neutrino Beam running for MiniBooNE was entirely in antineutrino mode. During this period, MiniBooNE collected data from $1.74 \times 10^{20}$ protons on target (POT). Added to previous antineutrino running, this brought MiniBooNE to a total of $6.8 \times 10^{20}$ protons on target in antineutrino mode. Antineutrino oscillation results with $5.66 \times 10^{20}$ were published in Phys. Rev. Lett. 105:181801 (2010). Figure 17 shows 99\% closed contours around the LSND preferred region, suggesting that in antineutrino mode MiniBooNE data are consistent with the earlier LSND results. More data is crucial to confirm or refute these results. 
MiniBooNE has been approved for up to $10 \times 10^{20}$ protons on target and has submitted a request to run for a total of $15 \times 10^{20}$ protons on target.

Figure 18 shows the integrated performance of the Booster Neutrino Beam for FY 2010. The performance of the Booster during FY 2010 was steady, with beam uptime of $85 \%$ and average weekly delivered protons of $0.35 \times 10^{19}$ protons on target/week. The weekly protons-ontarget spike at the end of FY 2010 was when the NuMI beam was down for its target replacement. MiniBooNE can take advantage of such unscheduled peak protons-on-target periods due to its high reliability and uptime. Also shown are various projections to the 2012 long shutdown for three proton/hour delivery rates. The actual rate is about $2.5 \times 10^{16}$ protons/hr (red line). At this rate, MiniBooNE should get close to the goal of $10 \times 10^{20}$ by the scheduled 2012 shutdown.

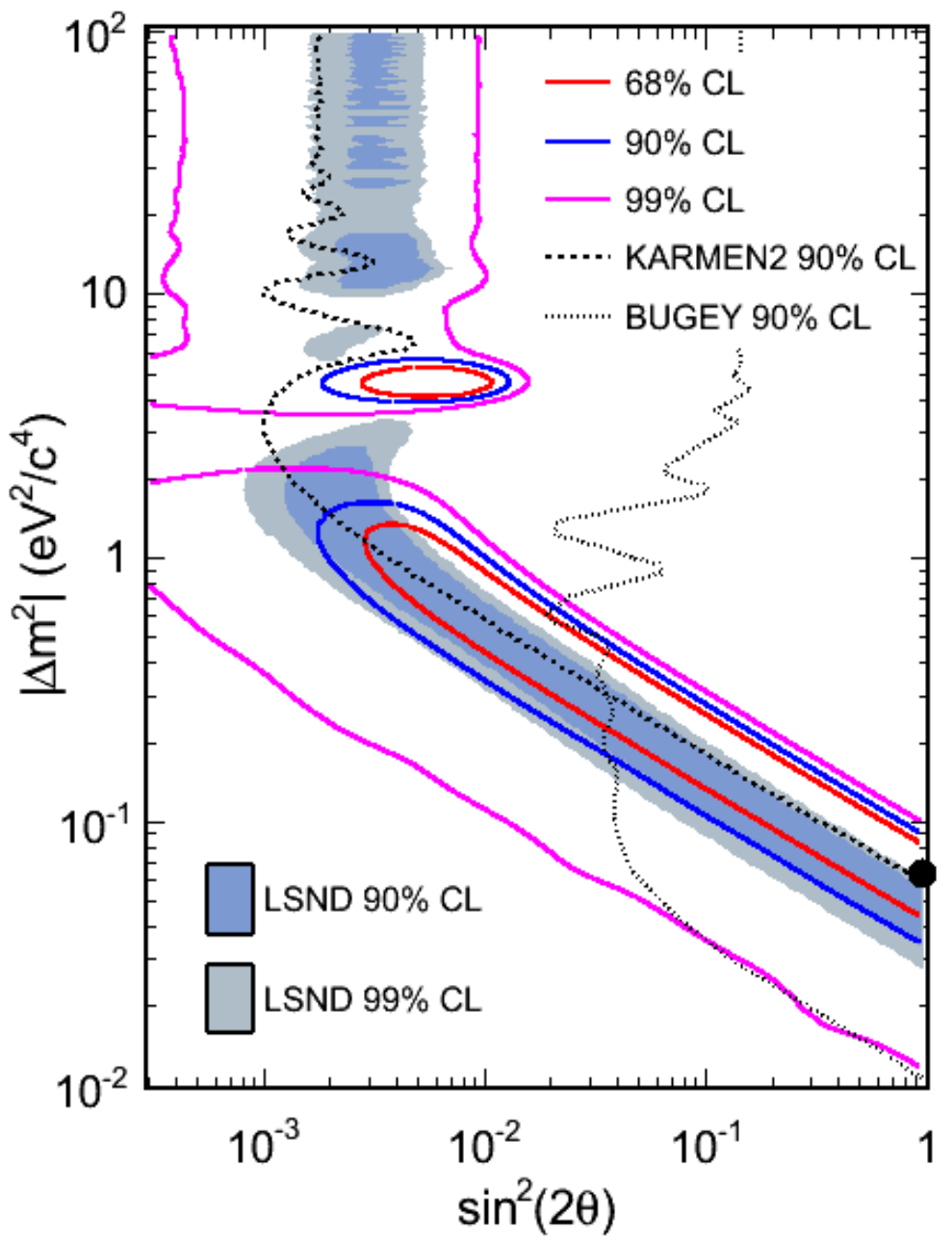

Figure 17. MiniBooNE 68\%, 90\%, and 99\% C.L. allowed regions within a two neutrino oscillation model from $5.66 \times 10^{20}$ POT for events with $\mathrm{E}_{\mathrm{QE}}>475 \mathrm{MeV}$. Also shown are limits from KARMEN and Bugey. The shaded areas show the 90\% and 99\% C.L. LSND allowed regions. The black dot shows the best fit point. 


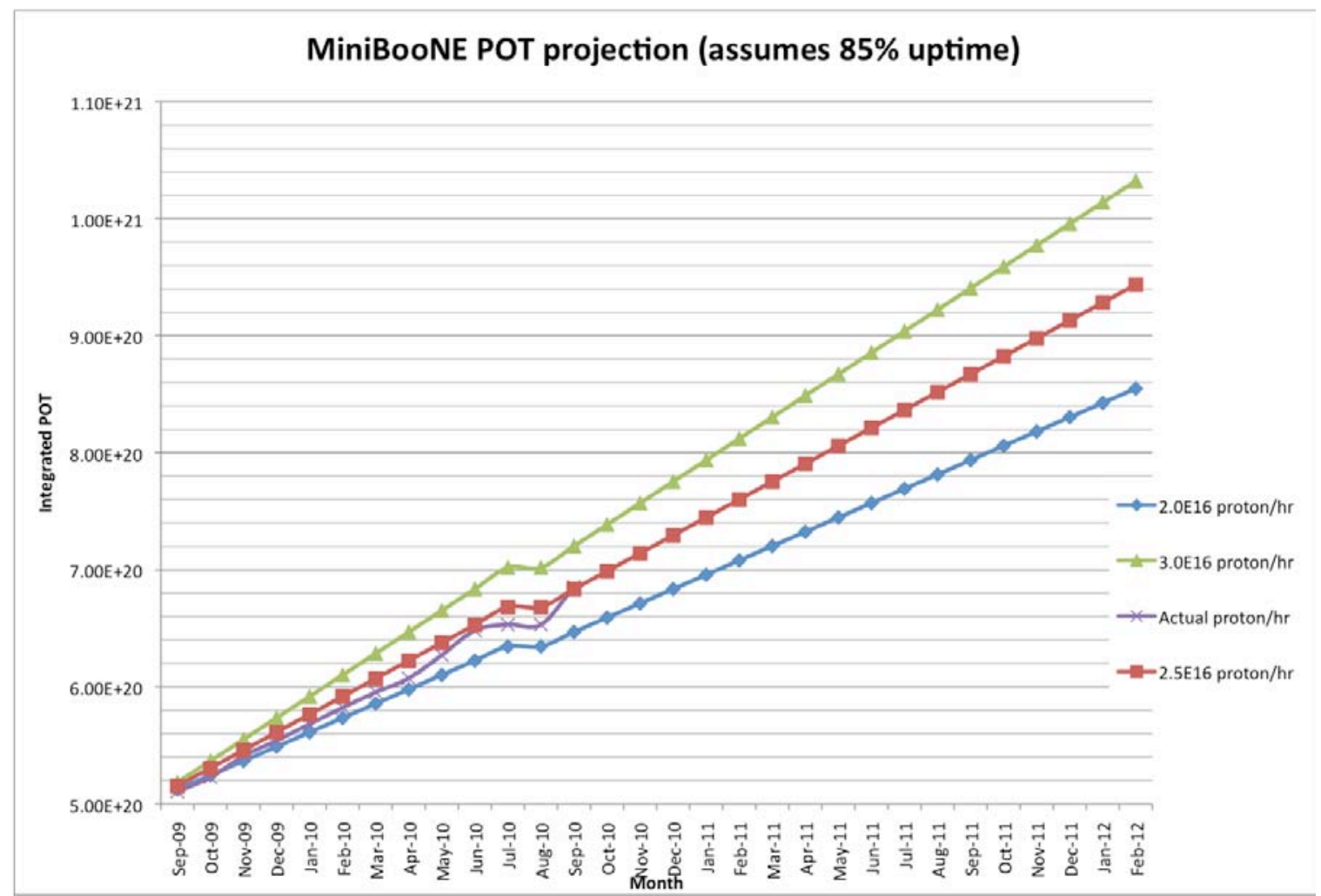

Figure 18. MiniBooNE actual integrated POT and projection for various beam rates. At the end of FY 2010 a total of $6.8 \times 10^{20}$ POT had been delivered in antineutrino mode. At historical rates of $2.5 \times 10^{16}$ protons/hr (red line) MiniBooNE should reach its goal of close to $10 \times 10^{20}$ POT by the long shutdown in early 2012.

Another important aspect of continued running of MiniBooNE is the reliability of the beamline and horn. For the FY 2010 antineutrino run period, the combined beamline and detector uptime was over 85\%. The most crucial element, the horn, operated for over 290 million pulses, surpassing the first horn, which failed six years ago with 94 million pulses. Both these numbers were world records at the respective times. This second horn recently developed a water leak and ground current trips. After a four week investigation during which the experiment was still able to run (which was during the high intensity period when NuMI was off), the source of the water leak was found and valved off. The ground current trips subsequently cleared up as well. At the end of the fiscal year, the horn was running with five of six water lines, but could lose a total of three before compromising horn cooling. In the unfortunate event of complete failure, a spare horn and target are available. It would require four weeks to install the replacement system.

The stability of the beamline and detector is demonstrated in Figure 19, which shows the number of neutrinos/POT over time. Aside from a short period with a beamline absorber problem in early 2006, the neutrino/POT rates have been constant. This clearly indicates that both the beamline and detector are stable over time. More detailed checks of neutrino energy response, efficiency, etc, show the same overall stability. 


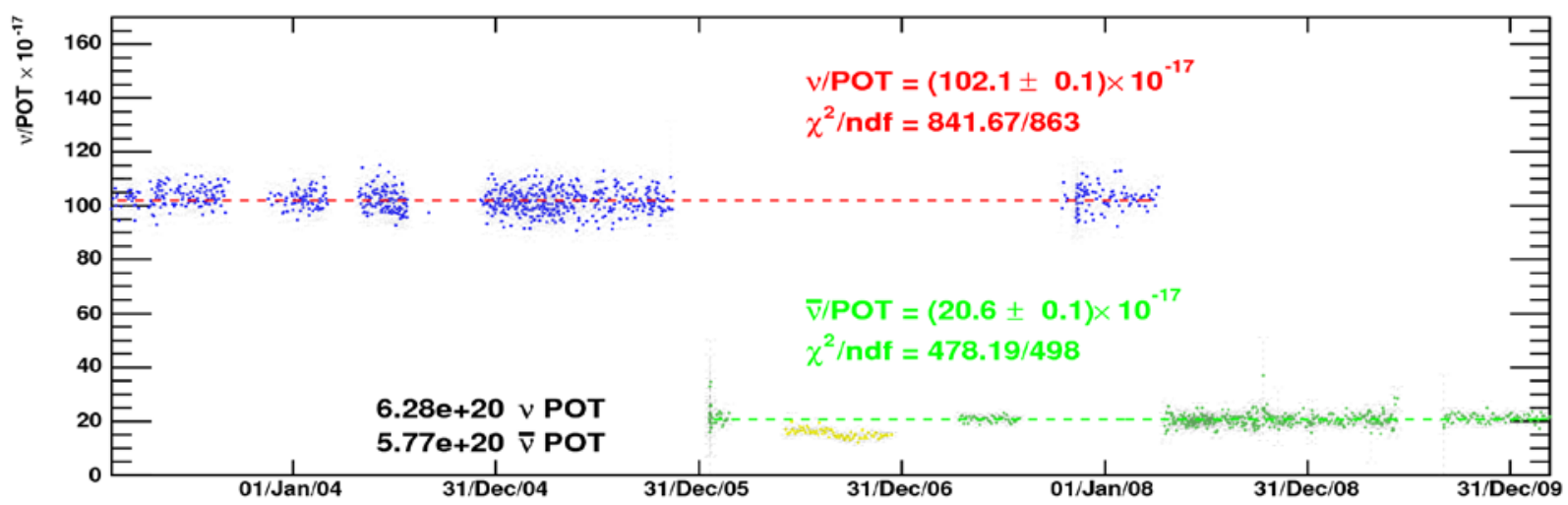

Figure 19. Neutrinos/POT for both neutrino and antineutrino running. The overall stability is excellent as shown by the $\mathrm{X}^{2}$ /ndf near one. The antineutrino mode does not include the absorber problem data in early 2006. The factor-of-five difference between neutrino and antineutrino modes is due to the reduced flux and cross section which makes taking high statistics antineutrino data samples difficult and time consuming.

Fixed-Target Switchyard 120 GeV (SY120) and MTest (A. K. Soha, R. Coleman, W. Kissel, C. Moore, E. Ramberg)

\section{Switchyard $120 \mathrm{GeV}$ Extraction from Main Injector in FY 2010}

The Fermilab test beam originates from the resonant extraction of one Booster batch inside the Main Injector (MI). This batch usually consists of 20-60 rf 'buckets', with buckets separated by 19 nsec. Thus the batch is about 1 microsecond long. The time required for a particle to transit the full circumference of the MI is about 11 microseconds. The batch is accelerated to $120 \mathrm{GeV}$, circulated around the MI, and is slowly extracted over a time period of a few seconds as a quadrupole gradually increases in field strength.

The length and duty cycle of the spill is determined by the Accelerator Division (AD), with guidance from the Program Planning Office. For FY 2010, there was a single 4-second-long spill per minute, for a maximum of up to 14 hours per day. The AD has set up a procedure for changing from this four-second spill to a one-second spill. The shorter spill can then be delivered more frequently for commissioning purposes, and for those groups which are limited by dataacquisition buffers. The number of SY120 pulses with beam in FY 2010 was 91,487 - over two and a half times the number in FY 2009.

In addition to the 4-second-long spill, a "ping" beam extraction from the Main Injector is available, in which the extraction quadrupole is brought close to its nominal strength, and then pulsed with current over a very short (1-5 msec) period. A large fraction of the beam then spills out in that short period. This can then be repeated at periodic intervals as the Main Injector holds its flattop. If the ping is $1 \mathrm{msec}$ long and the interval between pings is $200 \mathrm{msec}$, this replicates the macroscopic duty cycle of the proposed International Linear Collider (ILC). This makes Fermilab's MTest the only facility able to deliver high-energy particles with this structure. Figure 20 shows oscilloscope traces for this pinged extraction. 


\section{First Pings to MTest}
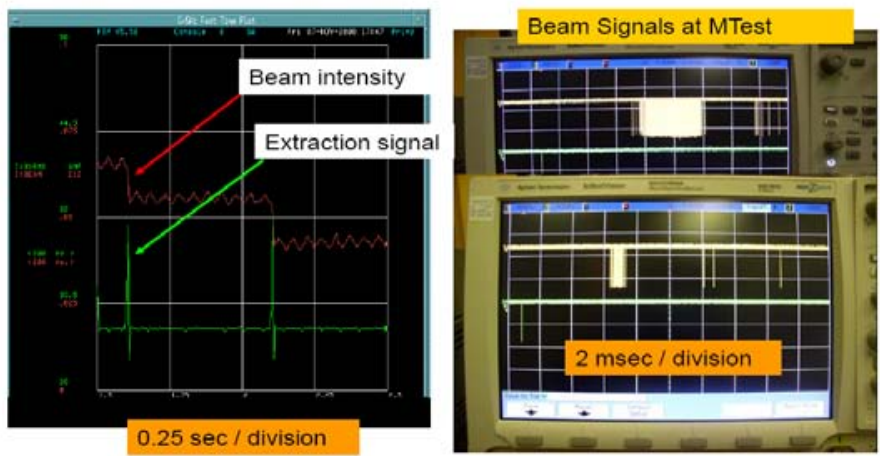

Figure 20. Two scope traces of scintillator hits during pinged MI extraction.

The extracted $120 \mathrm{GeV}$ primary proton beam has an approximate $0.3 \%$ momentum spread, and can be focused to a $7 \mathrm{~mm}$ rms spot size at the user facility (described below). In addition to delivering primary beam to users, the facility can target this primary beam on a secondary target and deliver lower-energy beams in the energy range of $1-66 \mathrm{GeV}$. There is a $1 \%$ to $10 \%$ momentum spread with approximately $2-5 \mathrm{~cm}$ rms spot size for secondary beams of lower momenta.

During 2007, the MTest secondary beamline was optimized for low-energy particle production. In addition to the ability to run at much lower energy, the new beamline had several other improvements in instrumentation and tuning facilities which improved general operations. The ILC CALICE calorimeter group (T-978) was particularly interested in the low-energy pion capability. In FY 2010, a number of groups utilized this secondary-beam mode. Tunes for the beamline were optimized for a variety of energies from 1 to $32 \mathrm{GeV} / \mathrm{c}$. In addition, it was observed that inserting a movable absorber located in the user area and tuning for positive 32 $\mathrm{GeV} / \mathrm{c}$ secondary beam maximizes the rate ( $\mathrm{kHz}$ level) of a wide-band muon beam.

\section{The Fermilab Test Beam Facility}

The Fermilab Test Beam Facility (FTBF) gives users from around the world an opportunity to set up their particle detectors in a variety of particle-beam types. A plan view of the facility is shown in Figure 14. The web-site URL for the facility is www-ppd.fnal.gov/FTBF. 


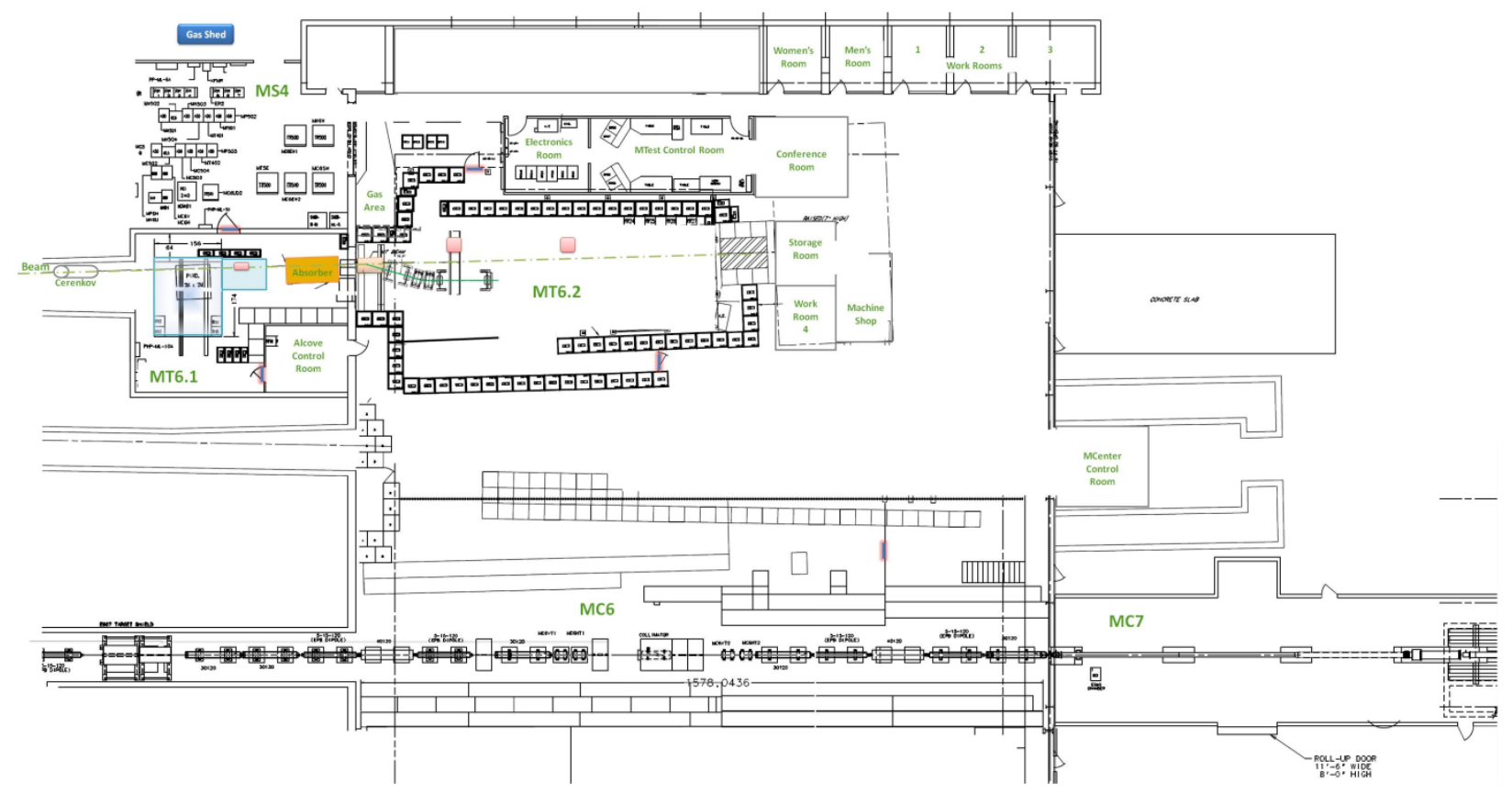

Figure 21. Plan view of the Fermilab Test Beam Facility.

\section{Research Performed at the FTBF in FY 2010}

Each test-beam experiment is required to prepare a Memorandum of Understanding with the Laboratory, in which the beam, infrastructure, and safety requirements are spelled out in detail. Five new experiments were approved and took data during FY 2010, and an additional six experiments returned from previous years to take more data in FY 2010. These eleven experiments are listed in Table 5 . The experiments installed equipment or took data for a total of 35 weeks, out of the 47 weeks with beam available during the year. In addition, there was about one week's time spread throughout the year devoted to facility beam studies.

Table 5. Test Beam experiments performed in FY 2010.

\begin{tabular}{|l|l|}
\hline Test & Description \\
\hline & \\
\hline T-1006 & Directly-Coupled Tiles (New) \\
\hline T-1005 & Muon g-2 Calorimeter Prototypes (New) \\
\hline T-1004 & Total-Absorption Dual-Readout Calorimetry R\&D (New) \\
\hline T-995 & Muon Detector/Tail Catcher R\&D (New) \\
\hline T-994 & JASMIN Radiation Measurements (New) \\
\hline T-992 & Radiation-Hard sensors for SLHC \\
\hline T-988 & AIRFLY - N2 Fluorescence \\
\hline T-979 & Ultra-Fast Timing \\
\hline T-977 & MINERvA Experiment Detector Calibration \\
\hline T-953 & U. Iowa Cerenkov Light Tests \\
\hline T-932 & Diamond Detector \\
\hline
\end{tabular}




\section{Developing a New Tertiary Low-Energy Beamline in the FTBF}

The MINERvA experiment (E-938) proposed installing an entirely new, tertiary beamline in the user facility to deliver $300 \mathrm{MeV} / \mathrm{c}$ pions onto their test apparatus (T-977). The installation of this beamline was begun in FY 2008 and was completed in the fall of 2009. A schematic drawing and photograph of the new beamline is shown in Figure 22. After completion of the MINERvA tests in spring 2010, this beamline became available for other users. The target and collimator can be rolled quickly aside so that the facility can also operate as before.
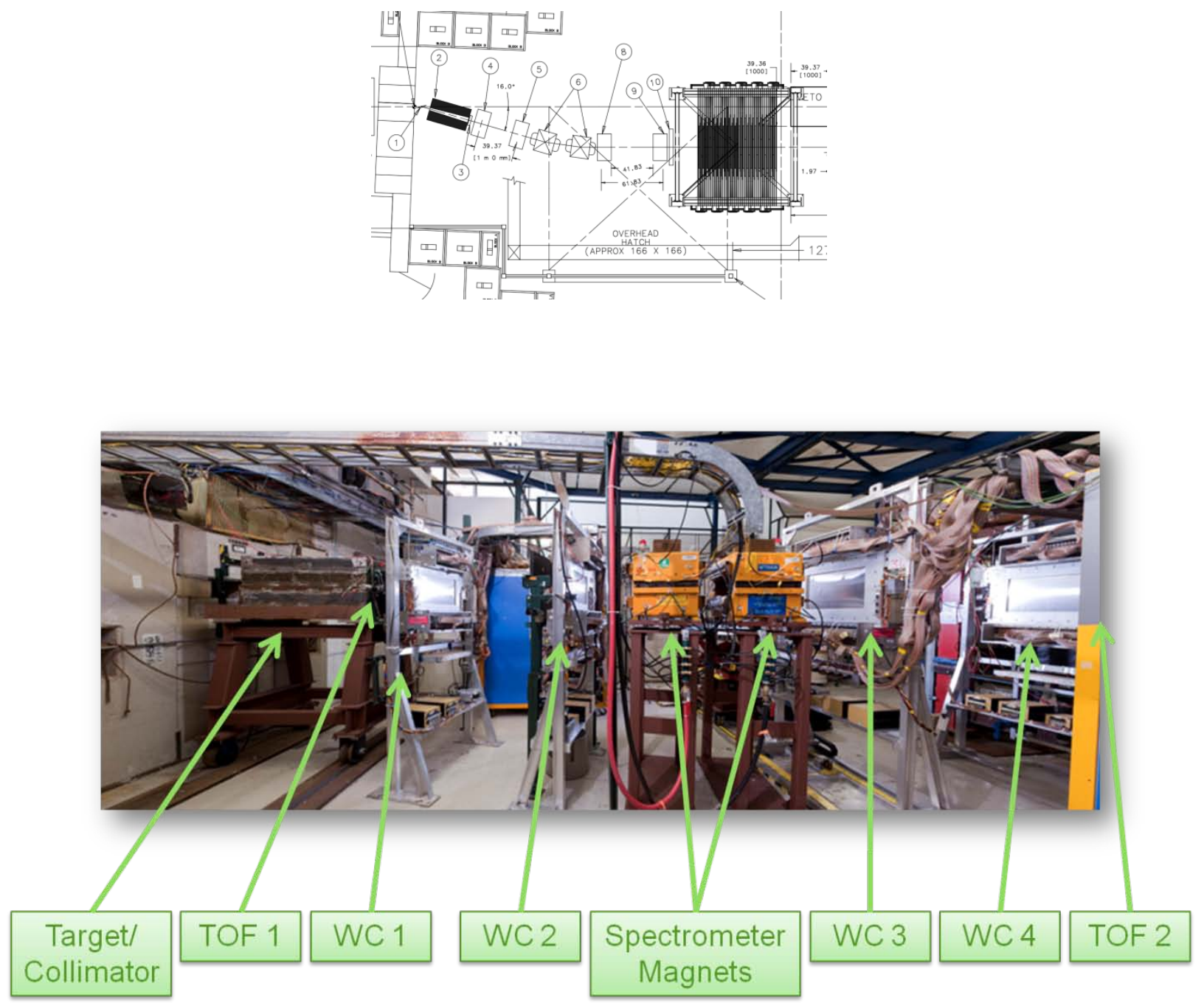

Figure 22. Schematic drawing and photo of the MINERvA tertiary beamline installation.

The T-977 beam tests completed during the summer at a nominal minimum momentum of $400 \mathrm{MeV}$, showed rates of about 200 particles per 4 second spill, of which $40 \%$ were protons and $60 \%$ pions (Figure 23). The beam momentum resolution dp/p was 3\%, multiple scattering limited for this momentum range. Wire chamber WC4 can be moved to achieve lower or higher momentum as desired, with a design momentum of $200 \mathrm{MeV}$ minimum. 


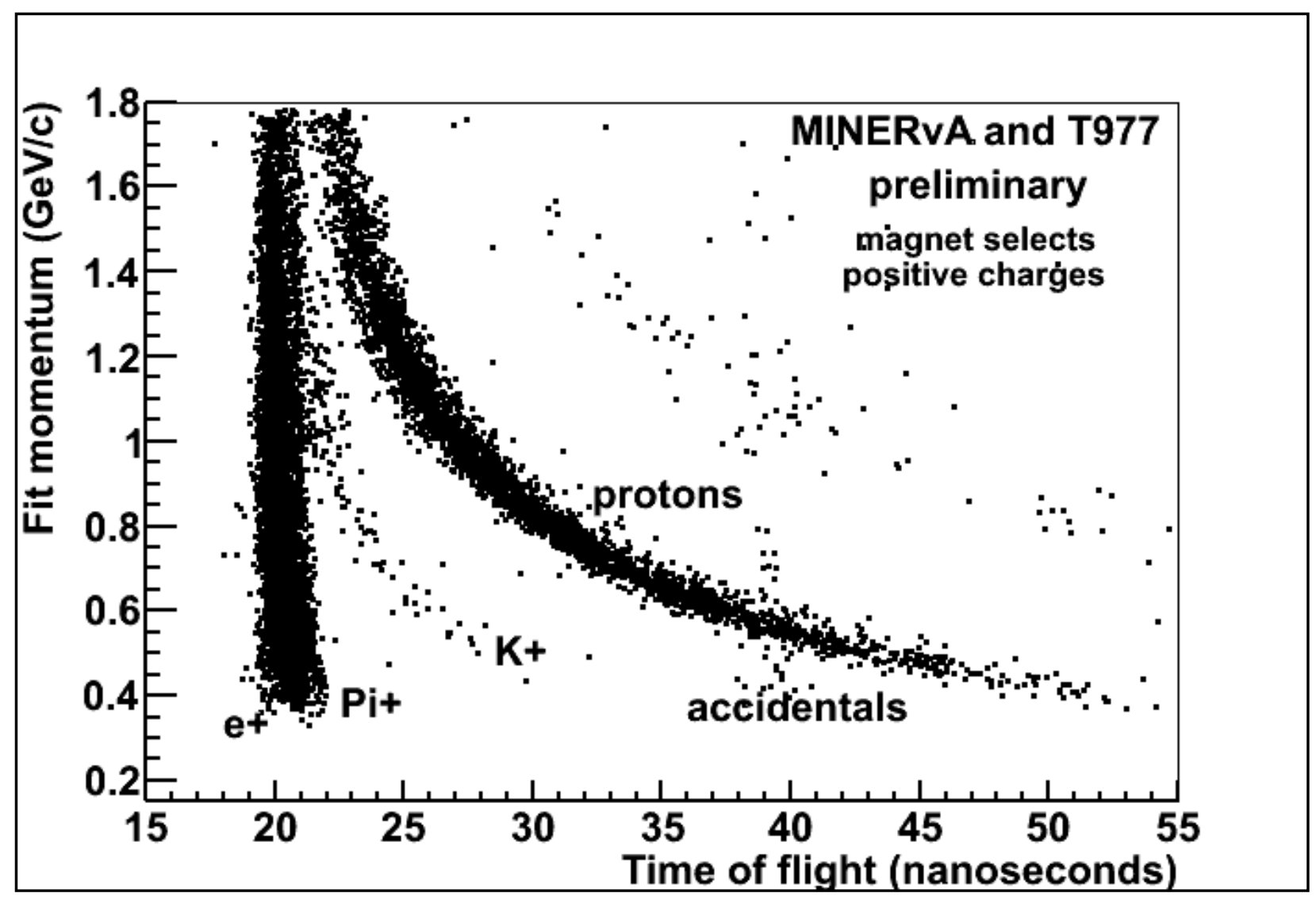

Figure 23. Momentum vs time of flight of tertiary particles produced at a nominal minimum $400 \mathrm{MeV}$ setting, showing the relative numbers and separation of the particles. 